\title{
„Wir kommen unter die Metzger.” Die Umsetzung des nationalsozialistischen Euthanasieprogramms im Reichsgau Tirol-Vorarlberg
}

\author{
Thomas Walli \\ Kerngebiet: Wirtschafts- und Sozialgeschichte \\ eingereicht bei: ao. Univ.-Prof. ${ }^{\text {in }}$ Dr. in Elisabeth Dietrich-Daum \\ eingereicht im Semester: SS 2014 \\ Rubrik: BA-Arbeit
}

\begin{abstract}
The National Socialist „Aktion T4" in the „Reichsgau Tirol-Vorarlberg"

The following bachelor thesis is about the "Aktion T4" in Nazi-Germany and its execution in the "Reichsgau Tirol-Vorarlberg". Starting with an overview of the most important ideological influences of the Nazis, like the social Darwinism or the theories about eugenics of the late $19^{\text {th }}$ century, it focuses on the statewide "Aktion T4". From 1939 on, the National Socialist regime aimed at killing all persons with a mental or physical handicap. One of the main hospitals in western Austria was the "Heil- und Pflegeanstalt Hall in Tirol". This paper examines the role of Hall within the "Aktion T4".
\end{abstract}

\section{Einleitung: Die Erbgesundheit als politisches Prinzip}

Spätestens seit dem Fund eines Gräberfeldes nahe der Heil- und Pflegeanstalt (kurz: HPA) Hall in Tirol rückte die historische Rolle der Psychiatrie im Kontext der nationalsozialistischen Euthanasiepolitik - und damit das grausame Euthanasieprogramm als solches - in den Fokus der öffentlichen Aufmerksamkeit. Insgesamt wurden 221 Skelette gefunden.' Ob die Verstorbenen der nationalsozialistischen „Rassenhygiene“ zum Op-

derStandard.at, Ausgrabungen in Hall in Tirol beendet, 14.11.2011, [http://derstandard.at/1319182805511/Psych iatrie-Friedhof-Ausgrabungen-in-Hall-in-Tirol-beendet], eingesehen 6.5.2014. 
fer fielen, ist noch nicht vollends geklärt und gilt es weiter zu untersuchen. Für die Nationalsozialisten war die Pflegeanstalt in Hall ein wichtiger Ort für die Umsetzung ihrer Euthanasiepolitik im Reichsgau Tirol-Vorarlberg. Sie diente während der ersten Jahre der "Aktion T4"2 - wie der systematische Massenmord an Menschen mit Behinderung im Nachhinein genannt wurde - in erster Linie als Sammelanstalt. Von hier aus wurden zahlreiche psychisch Kranke oder geistig behinderte Menschen in die Landesanstalt Hartheim oder in die Heil- und Pflegeanstalt Niedernhart bei Linz abtransportiert, wo sie ermordet wurden. Systematische Tötungen vor Ort in Hall in Tirol im Rahmen der sogenannten „wilden Euthanasie“ sind bislang nicht belegt. Die Erforschung der Jahre 1942 bis 1945 dauert noch an und dürfte mehr Licht ins Dunkel bringen.

Fest im sozialdarwinistischen und eugenischen Denken verwurzelt galten für die Nationalsozialisten Menschen mit körperlicher oder psychischer Behinderung im gesamten deutschsprachigen Gebiet als "minderwertig“. Zwangssterilisationen sollten dafür sorgen, dass sich "schwaches" oder "krankes Erbgut" nicht weiter in der Gesellschaft verbreitete. Die "Rassenhygiene” war zentraler Bestandteil der Politik der Nazis. Aber auch von außerhalb der Partei kamen bereits vor 1933 Forderungen, im Namen der Eugenik Sterilisationen und „Tötungen lebensunwerten Lebens“ durchzuführen. Dementsprechend waren Befürworter derartiger Maßnahmen vom neuen Reichskanzler Adolf Hitler und seiner Politik angetan: „Der Führer des Deutschen Reiches ist der erste Staatsmann, der die Erkenntnisse der Erbbiologie und "Rassenhygiene" zu einem bedeutenden Prinzip der Staatsführung gemacht hat,"4 meinte einer der damals führenden Rassenhygieniker, Otmar Freiherr von Verschuer, 1935. 1939 schließlich gab der „Führer" höchstpersönlich den für zigtausende Menschen verheerenden Befehl, die InsassInnen von Heil- und Pflegeanstalten zu erfassen und zu ermorden.

Das Programm zur Vernichtung von geistig kranken und körperlich behinderten Menschen war damit der erste systematische Massenmord der Nationalsozialisten. ${ }^{5}$ Das unter dem nichtssagenden Namen „Aktion T4" bekannt gewordene Euthanasieprogramm fand Anwendung im gesamten Deutschen Reich, so auch im Reichsgau TirolVorarlberg. Diese Arbeit widmet sich der Frage, wie die Planung, Vorbereitung und Umsetzung der NS-Euthanasie in besagtem Gau - und speziell in der Heil- und Pflegeanstalt Hall in Tirol - vonstattenging. Insgesamt werden die ideellen und theoretischen Grundlagen, an die sich die Nationalsozialisten anlehnten, die verschiedenen Phasen, die beteiligten Personen und die relevanten Orte der „Aktion T4" im Deutschen Reich und schließlich im Gau Tirol-Vorarlberg herausgearbeitet und systematisiert.

2 Die Bezeichnung „Aktion T4" findet sich in dieser Form in den zeitgenössischen Dokumenten nicht; sie hat sich erst nach 1945 etabliert. In den Quellen wird entweder von der „Aktion“ oder "Eu bzw. E-Aktion“ gesprochen; Brigitte Kepplinger, Tötungsanstalt Hartheim 1940-1945, in: Brigitte Kepplinger/Gerhart Marckhgott/Hartmut Reese (Hrsg.), Tötungsanstalt Hartheim (Oberösterreich in der Zeit des Nationalsozialismus 3), Linz 201333, S. 63-116, hier S. 67. In dieser Arbeit werden die Bezeichnungen "Aktion T4“, "Aktion“ und „T4" daher synonym verwendet. Wolfgang Neugebauer, Die „Aktion T4”, in: Kepplinger et al. (Hrsg.), Tötungsanstalt Hartheim, S. 19.

4 Otmar Freiherr von Verschuer in der Zeitschrift Der Erbarzt, Nr. 7/1935, zit. n. Ernst Klee, „Euthanasie“ im Dritten Reich. Die "Vernichtung lebensunwerten Lebens" (Die Zeit des Nationalsozialismus 18674), Frankfurt am Main 2010, S. 63.

$5 \quad$ Neugebauer, „Aktion”, S. 17. 
Insgesamt soll damit ein Bogen gespannt werden. Dieser beginnt mit dem dem nationalsozialistischen Regime übergestülpten ideologischen Überbau. Dessen Wurzeln und Ursprünge, wie der Sozialdarwinismus und die "Rassenhygienetheorien" des späten 19. Jahrhunderts, die Etablierung eben dieser Konzepte innerhalb der Wissenschaft während der Zeit der Weimarer Republik und deren Zusammenführung in der nationalsozialistischen Ideologie, werden dabei in den Blick genommen. Anschließend folgt die Darstellung der Politik der Nazis im Sinne dieser Ideologie und zum Zweck der Schaffung eines „reinen Menschengeschlechts“. Schließlich endet der dramaturgische Bogen bei der Darlegung der unmittelbaren Anwendung dieser politischen Maßnahmen in einem abgegrenzten Gebiet - nämlich dem Reichsgau Tirol-Vorarlberg. Damit sollen das Leid und der Wahnsinn greifbar gemacht werden, die ihren Ausgang in jenen wissenschaftlichen Konzepten fanden, die im Terrorregime des Dritten Reiches zu unumstößlichen Ideologien erklärt wurden.

Durch die Arbeit leitet folgende These: Auch der Reichsgau Tirol-Vorarlberg war an der Umsetzung der "rassenhygienischen" NS-Politik beteiligt. Insbesondere die HPA Hall in Tirol erfüllte dabei eine wichtige Funktion: Die Menschen wurden hier erfasst, gesammelt und selektiert, ehe sie in die Tötungsanstalt Hartheim abtransportiert wurden. Personell ist Dr. Hans Czermak, Leiter der staatlichen Gesundheitsverwaltung im Gau, neben Gauleiter Franz Hofer an erster Stelle zu nennen. Im Rahmen der HPA Hall war Primar Dr. Ernst Klebelsberg eine der zentralen Akteure: Er konnte aktiv an den Selektionen mitwirken und machte sich damit mitschuldig am Tod der abtransportierten AnstaltsinsassInnen.

Zunächst wird die Idee der "Rassenhygiene" und der Euthanasie mit der Rassenideologie der Nationalsozialisten in Verbindung gebracht und dargestellt. Im dritten Kapitel wird die "Aktion" skizziert: ihr Ursprung, von der Implementierung der ersten Gesetze zur Zwangssterilisation bis zu Hitlers Befehl zur Tötung von Menschen mit Behinderung, ihre Umsetzung und die relevanten Personen, Orte und Methoden. Dies soll die Vorarbeit darstellen zu der Beantwortung der Frage nach der Umsetzung der Euthanasiepolitik in Tirol und Vorarlberg, die Inhalt von Kapitel vier ist. Hier werden wiederum die Vorbereitung, die Durchführung anhand der Darstellung der einzelnen Transporte in die Anstalten Hartheim und Niedernhart und der Reaktionen - soweit bekannt - von Opfern und Verwandten und der Widerstand dagegen thematisiert. Zum Schluss wird die Frage gestellt, was sich nach dem Ende der offiziellen „T4“ im Gau Tirol-Vorarlberg und vor allem in der HPA Hall in Tirol abspielte.

Die für diese Arbeit relevante Literatur ist eine Kombination aus älteren Werken der 1980er-Jahre, wie etwa Hans-Walter Schmuhls "Rassenhygiene, Nationalsozialismus; Euthanasie. Von der Verhütung zur Vernichtung ,lebensunwerten Lebens', 1890-1945" von 1987, sowie aktuellen Werken (siehe weiter unten). Schmuhls Werk stellt trotz seines Alters ein Standardwerk für die wissenschaftliche Beschäftigung mit diesem Thema dar.

Florian Leimgrubers erstaunlich umfassende Dissertation „Euthanasie und Sterilisierung im ehemaligen ,Reichsgau Tirol-Vorarlberg' während des II. Weltkrieges 1939-45" 
(1988) wurde insbesondere für das zweite Kapitel herangezogen. Seine Bedeutung für Kapitel vier hält sich aufgrund der neuen Erkenntnisse der letzten Jahre leider in Grenzen. Für bestimmte Stellen in Kapitel zwei wurde ebenfalls Peter Emil Beckers „Zur Geschichte der Rassenhygiene. Wege ins Dritte Reich“ herangezogen. Das Werk sollte aber mit Vorsicht betrachtet werden, da Becker einst selbst Mitglied der SA und der NSDAP war. In seiner Entnazifizierungsakte wurde er als "Mitläufer" deklariert. ${ }^{6}$

Ein weiteres Standardwerk, das trotz enormer Fülle an Informationen sehr kompakt wirkt, ist Ernst Klees „Euthanasie' im Dritten Reich. Die ,Vernichtung lebensunwerten Lebens" von 2010. Es handelt sich um eine vollständig überarbeitete Neuausgabe seines bekannten Buches „'Euthanasie' im NS-Staat" von 1985. Ein weiteres Grundlagenwerk aus den 1980er-Jahren ist „Rasse, Blut und Gene. Geschichte der Eugenik und Rassenhygiene in Deutschland" von Peter Weingart, Jürgen Kroll und Kurt Bayertz. Die Darstellung der NS-(Rassen-) Ideologie darin ist sehr umfassend.

Für Kapitel drei und vier ist neben Klee und Schmuhl vor allem der Sammelband "Tötungsanstalt Hartheim" (3. Auflage 2013) relevant. Die darin befindlichen Artikel zu unterschiedlichen Themen in Bezug auf die "Aktion T4" vor allem in Österreich sind übersichtlich, sehr gut recherchiert und auf aktuellsten Stand. Besonders Oliver Seiferts Beiträge über die HPA Hall in Tirol, Brigitte Kepplingers Beiträge zu der Anstalt Hartheim und dem Ablauf von "T4" in Österreich sowie Wolfgang Neugebauers Beitrag zur "Aktion“ waren für das Zustandekommen dieser Arbeit von unschätzbarem Wert. Nicht weniger bedeutend war der Sammelband der "Kommission zur Untersuchung der Vorgänge um den Anstaltsfriedhof des Psychiatrischen Krankenhauses in Hall in Tirol in den Jahren 1942 bis 1945". Die darin befindlichen Artikel wiederum von Seifert, Kepplinger und Neugebauer sowie von Elisabeth Dietrich-Daum, Dirk Dunkel, George McGlynn/Nadine Carlichi-Wijes und Friedrich Stepanek enthalten die aktuellsten Informationen rund um das Thema und sind für diese Arbeit unverzichtbar. Schließlich sei noch Horst Schreibers Werke genannt, der mit "Nationalsozialismus und Faschismus in Tirol und Südtirol. Opfer, Täter, Gegner" ein Standardwerk für diese Epoche im Tiroler Raum schuf und dessen Artikel über Dr. Hans Czermak einen guten Einblick in die Psyche und persönliche Geschichte eines NS-Täters gibt.

\section{„Was nicht gute Rasse ist auf dieser Welt, ist Spreu“:? Die nationalsozialistische Ideologie der „Rassenhygiene“ und deren Ursprung}

\section{Sozialdarwinismus}

Charles Darwin veröffentlichte sein Hauptwerk "The Origin of Species by Means of Natural Selection, or the Preservation of Favoured Races in the Struggle for Life" im November 1859 in London. Es sollte den Grundstein legen für eine neue Auffassung

6 Entnazifizierungsakte Dr. Peter Emil Becker, 9.3.1948, Staatsarchiv Sigmaringen (Landesarchiv Baden-Württemberg), Bestand Wü 13 T 2: Staatskommissariat für die politische Säuberung, [https://www2.landesarchiv-bw.de/ ofs21/olf/struktur.php?bestand=593\&sprungld=3232265\&letztesLimit=suchen], eingesehen 7.9.2014. 
über das Leben, die Entwicklung des Lebens auf der Erde und in weiterer Folge die Gesellschaft als einen sich evolutionär entwickelnder Organismus. Darwin ließ den Menschen zunächst außen vor, doch dauerte es nicht lange, bis seine Theorie auch in sozialphilosophischen und -politischen Diskursen Eingang fand. Die "Sonderstellung" des Menschen stellte er selbst zwölf Jahre später allerdings in Frage, als er die Entwicklung des Menschen als Bestandteil des gesamten Evolutionsprozesses ansah. ${ }^{8}$

Spätestens in den 1880er-Jahren galt der Darwinismus als gefestigt und allgemein anerkannt. Zur gleichen Zeit gab es auch die ersten tiefer gehenden Überlegungen zum Sozialdarwinismus, i. e. die wissenschaftlich nicht haltbare Anwendung der Theorien und Konzepte Darwins auf die Gesellschaft(en), ${ }^{9}$ mit anderen Worten "die Übertragung der Gültigkeit von Darwins Prinzipien auf den sozialen Bereich".10 Zentral für Darwin und später den Sozialdarwinismus sind Formeln wie der "Kampf ums Dasein", die „natürliche Auslese“ und das „Überleben des Stärksten“, wobei letztere auf eine (unglücklich) gewählte Übersetzung des Ausspruches „survival of the fittest ${ }^{\prime 11}$ von Darwin zurückgeht. ${ }^{12}$

\section{Eugenik}

Einer der ersten, der aus den Lehren der Evolutionstheorie sozialpolitische Maßnahmen ableitete, war Darwins Vetter, der Arzt und Anthropologe Francis Galton. Dieser gilt als Begründer des Begriffs „Eugenik",13 den er 1883 das erste Mal verwendete. Dabei theoretisierte er sowohl die positive Eugenik, also die Vermehrung der Erbanlagen von „Tüchtigen", als auch die negative Eugenik, die Verminderung von Erbanlagen, die als negativ betrachtet wurden. ${ }^{14}$ Einige der von ihm geforderten Maßnahmen wurden in den folgenden Jahrzehnten in den Vereinigten Staaten von Amerika umgesetzt: Zum Beispiel verschiedene Sterilisierungsgesetze, die seit 1907 in mehreren Bundesstaaten (darunter beispielsweise Kalifornien) eingeführt wurden. ${ }^{15}$ In Deutschland waren Otto Ammon und Ernst Haeckel Wegbereiter des Sozialdarwinismus eugenischer Ausprägung. So äußerte sich beispielsweise der Rassenanthropologe Ammon in Bezug auf "mißratene [sic!] Individuen", sprich Menschen mit Behinderung, dahingehend, dass die "Verhinderung ihrer Fortpflanzung"16 von wichtigem Gesellschaftsinteresse sei. Er

8 Florian Leimgruber, Euthanasie und Sterilisierung im ehemaligen „Reichsgau Tirol-Vorarlberg“ während des II. Weltkrieges 1939-45, Diss. Innsbruck (1988), S. 57 f.

9 Horst Schreiber, Nationalsozialismus und Faschismus in Tirol und Südtirol. Opfer, Täter, Gegner (Tiroler Studien zu Geschichte und Politik 8), Innsbruck 2008, S. 209.

10 Peter Emil Becker, Zur Geschichte der Rassenhygiene. Wege ins Dritte Reich, Stuttgart, New York 1988, S. 8.

11 Heute spricht man deshalb eher vom Überleben des am besten Angepassten.

12 Klee, Euthanasie, S. 19

13 Der Begriff stammt vom griechischen eugenếs und meint so viel wie „wohlgeboren“, „Von edler Abkunft“; ,Eugenik”, in: Duden, online [http://www.duden.de/node/685055/revisions/1262418/view], eingesehen 8.9.2014.

14 Klee, Euthanasie, S. 19

15 Hans-Walter Schmuhl, Rassenhygiene, Nationalsozialismus, Euthanasie. Von der Verhütung zur Vernichtung „lebensunwerten Lebens", 1890-1945 (Kritische Studien zur Geschichtswissenschaft 75), Göttingen 1987, S. 30.

16 Otto Ammon, Die Gesellschaftsordnung und ihre natürlichen Grundlagen. Entwurf einer Sozial-Anthropologie zum Gebrauch für alle Gebildeten, die sich mit sozialen Fragen befassen, Jena 1896², S. 249. 
lehnte eine „künstliche Zuchtwahl“ ab, mit der Begründung, dass der Mensch nicht die Aufgabe der Natur übernehmen dürfe. ${ }^{17}$

Ernst Haeckel, seines Zeichens Zoologe, verlangte bereits 1863 in seiner Jungfernrede vor der Versammlung Deutscher Naturforscher und Ärzte in Stettin, die evolutionsbiologischen Theorien Darwins auf die Menschen zu übertragen, zu einer Zeit, als die Evolutionstheorie noch keineswegs als gesichert galt. ${ }^{18}$ Im Sinne der Eugenik machte Haeckel unter anderem auf die "contraselektorischen" Effekte in der Medizin aufmerksam. Durch medizinische Eingriffe würden, so Haeckel, Erbkrankheiten innerhalb der Gesellschaft zunehmen. Gleichzeitig stellte er diese Effekte auch in der Tradition der spartanischen Kindestötungen ${ }^{19}$ dar, die er als einen "Ausdruck bewußter [sic!] Erbpflege "20 deutete und wofür er nur positive Worte fand: "Gewiß [sic!] verdankt das Volk von Sparta dieser künstlichen Auslese oder Züchtung zum großen Theil [sic!] den seltenen Grad von männlicher Kraft und rauher [sic!] Heldentugend, durch die es in der alten Geschichte hervorragt."21 Dass Haeckel über dies hinaus nicht explizit Kindestötungen forderte, ist nur auf den ersten Blick erstaunlich. Denn es lag schlichtweg an Haeckels Interesse an der natürlichen Auslese und damit seiner Abneigung gegenüber politischen Maßnahmen, die ihn von Forderungen nach Euthanasie absehen ließen. Er glaubte an ein Ziel, auf das sich die Natur als Ganzes hinbewege, und dass es nicht nötig sei, sich in diesen Prozess einzumischen. Erst mit dem Ende dieser teleologischen Sichtweise und dem Aufkommen eines größeren Kulturpessimismus sowie einer umfassenderen Zivilisationskritik gewann der Euthanasiegedanke ${ }^{22}$ mehr Bedeutung innerhalb der Eugenik. ${ }^{23}$

\section{"Rassenhygiene"}

So etablierte sich in Deutschland - neben den vorrangig britischen Eugenikern jener Zeit - gegen Ende des 19. Jahrhunderts eine Gruppe von besonders eifrigen Sozialdarwinisten. Es handelte sich um deutsche Ärzte, Biologen und Anthropologen, die als Vertreter der "Rassenhygiene" auftraten und diese salonfähig machten. Zu den bedeutendsten unter ihnen zählen Alfred Ploetz, von dem der Begriff der "Rassenhygiene" stammt, Wilhelm Schallmayer, Fritz Lenz, Christian Freiherr von Ehrenfels sowie die in ihren Ansichten radikalsten Schriftsteller Ernst Mann und Alexander Tille. Dabei waren

17 Schmuhl, Rassenhygiene, S. 32.

18 Ebd., S. 31.

19 In der Forschung ist man sich weitestgehend einig, dass die spartanischen Kindestötungen, die auf Schilderungen Plutarchs zurückgehen (beschrieben in Plutarch, Vita Lycurgii XVI.), nichts als eine Legende darstellen; Klee, Euthanasie, S. 606.

20 Zit. n. Schmuhl, Rassenhygiene, S. 32.

21 Ernst Haeckel, Natürliche Schöpfungsgeschichte. Gemeinverständliche wissenschaftliche Vorträge über die Entwicklungslehre im Allgemeinen und diejenige von Darwin, Goethe und Lamarck im Besonderen, über die Anwendung derselben, auf den Ursprung des Menschen und andere damit zusammenhängende Grundfragen der Naturwissenschaft, Berlin 1870², [http://babel.hathitrust.org/cgi/pt?id=hvd.32044066291840;view=1 up;seq=11], eingesehen 7.10.2014, S. 152.

22 Der Begriff „Euthanasie" entstammt dem griechischen euthanasía, das so viel wie "leichter Tod" bedeutet; ,Euthanasie, in: Duden, online [http://www.duden.de/node/685056/revisions/1193702/view], eingesehen 9.9.2014.; vertiefend zum Begriff und dessen vielseitigen Bedeutungen: Schmuhl, Rassenhygiene, S. 25-28. 
die Rassenhygieniker ohnehin in ihren Ansichten extremer als ihre angelsächsischen Kollegen. So fanden beispielsweise die Mittel der negativen Euthanasie Eingang in ihre Theorien. Überhaupt wurde das Euthanasieprinzip integraler Bestandteil ihrer Werke. ${ }^{24}$ Im Folgenden werden die Gedanken und rassischen Konzepte von einigen wenigen Exponenten der deutschen „Rassenhygiene“ dargestellt.

\section{Wilhelm Schallmayer}

Der Arzt Wilhelm Schallmayer verfasste um die Jahrhundertwende mehrere Werke über eugenische Themen. Als sein Hauptwerk gilt „Vererbung und Auslese im Lebenslauf der Völker, eine staatswissenschaftliche Studie aufgrund der neueren Biologie", das als dritter Teil der Preisschriftensammlung "Natur und Staat", herausgegeben von Heinrich Ernst Ziegler (deutscher Zoologe), Johannes Conrad (Nationalökonom) und Ernst Haeckel, 1903 in Jena publiziert wurde. Es erschien in immer neueren Auflagen und avancierte zum Standardwerk der Eugenik in Deutschland. In diesem und in anderen Schriften erörtert Schallmayer, dass sich die erblichen Eigenschaften - insbesondere die Leistungsfähigkeit - von Gesellschaften immer wieder in einem zeitlich langen Prozess veränderten. Seine Grundidee ist dabei so simpel wie folgenschwer: Eine Gesellschaft setzt sich aus verschiedenen Personen zusammen, mit jeweils verschiedenen Erbanlagen; somit sind die einzelnen Personen einer Gesellschaft in puncto „Rassetüchtigkeit“ ungleich. Gleichzeitig „vermehren“ sich nicht alle Personen genau gleich, was bedeutet, dass sich manche Erbanlagen mehr verbreiten als andere. ${ }^{25} \mathrm{Als}$ Folge resultiert für den Staat die Notwendigkeit einer „zweckmäßigen Beeinflussung des sozialgenerativen oder Rasseprozesses sowohl in quantitativer als auch in qualitativer Hinsicht“26. Das heißt mit einfachen Worten, der Staat solle für eine Vermehrung der Erbanlagen, die zu einem Ansteigen der "Rassetüchtigkeit" führen, sorgen. Dabei schweben Schallmayer weniger Gesetze im Bereich der Ehe und Fortpflanzung vor, als vielmehr "Beeinflussungen der öffentlichen Meinung im Sinne des Rassedienstes",27 Reformen im Bereich der Kinderförderung, Sexualordnung, des Erbrechts, des Steuerwesens, des Sanitätswesens etc. Als besonders wichtig sah er die Einführung von sogenannten „obligatorischen Personalbögen“ an. ${ }^{28}$ Diese sollten eventuelle Krankheiten, genetische Anomalien und in einem weiteren Schritt einen kurzen Stammbaum enthalten. So hätte der Staat Eheverbote unter Bezugnahme auf die "Rassetüchtigkeit" verordnen können. Die Einführung derartiger Bögen erlebte Schallmayer nicht mehr, aber in dieser Tradition standen mehrere Maßnahmen der Nationalsozialisten, wie beispielsweise die "Nürnberger Gesetze".29

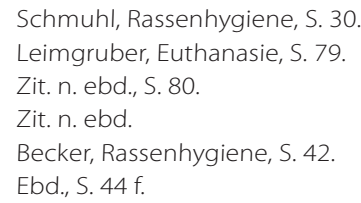


Nach Schallmayers Tod 1919 gerieten er und sein Werk zunehmend in Vergessenheit. Seine Wirkung auf und Bedeutung für die nachkommenden Rassenhygieniker darf jedoch nicht unterschätzt werden. ${ }^{30}$

\section{Alfred Ploetz}

Der Begriff „Rassenhygiene“ ist mit seinem Schöpfer, Alfred Ploetz, untrennbar verbunden. Dieser veröffentlichte 1895 sein Hauptwerk „Die Tüchtigkeit unserer Rasse und der Schutz der Schwachen. Grundlinien einer Rassen-Hygiene". Dabei verwendete Ploetz die Bezeichnungen Eugenik und "Rassenhygiene" noch als Synonyme. ${ }^{31}$ Ploetz hatte sein Leben ganz der "Rassenhygiene" verschrieben; er sah darin sein Lebenswerk, eine Art der "religiösen Offenbarung“.32 Bereits in Studentenjahren gründete er zusammen mit einigen Freunden (darunter auch Autor Gerhart Hauptmann) den Verein "Pacific", der sich das Ziel setzte, „eine Art Kolonie [...] auf freundschaftlicher, sozialistischer und wohl auch pangermanischer Grundlage ${ }^{\prime 33}$ in einem Pazifikstaat zu errichten. Um Ideen für einen derartigen Staat zu sammeln, entschied sich Ploetz einige Zeit in der Kolonie Ikarien in den Vereinigten Staaten zu verbringen. Hier hatte der französische Sozialist Étienne Cabet eine nach sozialistischen Prinzipien geleitete Dorfgemeinschaft errichtet. Nach sechs Monaten kehrte Ploetz enttäuscht zurück. Durch die Erfahrung des Misserfolges von Cabets Experiment kam er zur fatalen Erkenntnis, „daß [sic!] mit dem heutigen durchschnittlichen Menschenmaterial der Zusammenhalt solcher Kolonien [...] nicht aufrechtzuerhalten wäre".34

Ploetz' Forderungen waren denkbar hart: Nur Paare mit ausgesprochen guten Erbanlagen durften Kinder in die Welt setzen. Dem Staat komme die Aufgabe zu, die Fortpflanzung dahingehend zu reglementieren. ${ }^{35}$ Dabei schwebten Ploetz nicht nur Mittel der positiven Eugenik vor; die „Ausjätung”, das heißt die (Kinder-) Euthanasie, war ein legitimes Instrument des Staates. Gleichzeitig sollte einer „Kontraselektion“ vorgebeugt werden, bei der (beispielsweise durch Kriege) gerade diejenigen vernichtet würden, deren Erbmasse als qualitativ gut gelte, und jene (teuer) am Leben blieben, die ein in Ploetz' Augen "schlechtes" und "schwaches" Erbgut besaßen. ${ }^{36}$ Hilfe für Arme und Schwache sind für inn nichts als Gefühlsduselei:

„Solche [die Armenfürsorge, T. W.] und andere,humane Gefühlsduseleien' wie Pflege der Kranken, der Blinden, der Taubstummen, überhaupt aller Schwachen, hindern oder verzögern nur die Wirksamkeit der natürlichen Zuchtwahl. [...] Der Kampf ums Dasein muss in seiner vollen Schärfe erhalten bleiben." ${ }^{137}$

Leimgruber, Euthanasie, S. 78.

Becker, Rassenhygiene, S. 58; Klee, Euthanasie, S. 19.

Becker, Rassenhygiene, S. 58.

Ebd., S. 59.

Alfred Ploetz, Lebenserinnerungen (im Ploetz-Archiv), zit. n. ebd., S. 60.

Klee, Euthanasie, S. 19.

Leimgruber, Euthanasie, S. 84

Alfred Ploetz, Grundlinien einer Rassenhygiene, Nachdruck von Weß, S. 98, zit. n. Klee, Euthanasie, S. 20. 
Alfred Ploetz, immer nur das Ziel der vollkommen erbgesunden Rasse vor Augen, gründete 1904 ein Periodikum mit dem etwas sperrigen Namen „Archiv für Rassen- und Gesellschafts-Biologie einschließlich Rassen- und Gesellschafts-Hygiene. Zeitschrift für die Erforschung des Wesens von Rasse und Gesellschaft und ihres gegenseitigen Verhältnisses, für die biologischen Bedingungen ihrer Erhaltung und Entwicklung, sowie für die grundlegenden Probleme der Entwicklungslehre". Interessant in diesem Zusammenhang ist, dass ab Januar 1905 der Psychiater und Schwager von Ploetz, Ernst Rüdin, die Texte für die Veröffentlichung im Heft redigierte. Klee schreibt dabei zu Recht von einer "Ehe mit Symbolcharakter:, Rassenhygiene' und Psychiatrie verschwägern sich"38. Später ließen sich Pauline Rüdin, Ernst Rüdins Schwester, und Alfred Ploetz wegen Kinderlosigkeit einvernehmlich scheiden. ${ }^{39}$

Neben dieser Zeitschrift machte Ploetz zu Beginn des 20. Jahrhunderts vor allem mit der Gründung der "Gesellschaft für Rassenhygiene” (ab 1914 „Deutsche Gesellschaft für Rassenhygiene", DGR) auf sich aufmerksam. Mitbegründer und spätere Mitglieder sind unter anderem der Botaniker Erwin Baur, der Rassenforscher Eugen Fischer, der Erbbiologe Fritz Lenz, der sozialdemokratische Hygieniker Alfred Grotjahn, der zusammen mit dem ersten Vorsitzenden Max von Gruber maßgeblich für die rasche Anerkennung der Bewegung in Deutschland verantwortlich war, sowie der Schriftsteller Gerhart Hauptmann. Andere Vereinigungen mit ähnlich klingenden Namen folgten: so zum Beispiel die "Gesellschaft für soziale Medizin, Hygiene und Medizinalstatistik", die im gleichen Jahr (1905) gegründet wurde, oder in Österreich die „Wiener Gesellschaft für Rassenpflege (Rassenhygiene)" (WGR) ${ }^{40}$ von 1925.41

\section{Gedanken über das „lebensunwerte Leben“ und der „Baur-Fischer-Lenz”}

Zwei Jahre nach Ende des Ersten Weltkrieges und des "Schandfriedens von Versailles" gaben der hochrangige Jurist Karl Binding und der Direktor der Freiburger Universitätsklinik Alfred Erich Hoche ein zweibändiges, 62 Seiten umfassendes Büchlein mit dem Titel "Die Freigabe der Vernichtung lebensunwerten Lebens. Ihr Maß und ihre Form” heraus. Die Schrift gliedert sich in zwei Teile. Im ersten Teil, von Binding verfasst, findet sich eine Gegenüberstellung von im Krieg oder in Bergwerken getöteten gesunden jungen Männer mit den InsassInnen von Heil- und Pflegeanstalten. Getötet werden dürften laut dem Autor alle „unheilbar Blödsinnigen": „Ihr Leben ist absolut zwecklos [...]. Ihr Tod reißt nicht die geringste Lücke - außer vielleicht im Gefühl der Mutter oder

38 Alfred Ploetz, Grundlinien einer Rassenhygiene, Nachdruck von Weß, S. 98, zit. n. Klee, Euthanasie, S. 20.

39 Ebd., S. 606.

40 In Österreich waren es vor allem deutschnational bzw. völkisch geprägte Vereinigungen, die zwischen 1923 und 1926 gegründet wurden. Neben der genannten WGR waren die beiden bedeutendsten die Oberösterreichische Gesellschaft für Rassenhygiene in Linz sowie die Grazer Gesellschaft für Rassenhygiene. Alle nahmen die DGR als Vorbild; Thomas Mayer, Familie, Rasse und Genetik. Deutschnationale Eugeniken im Österreich der Zwischenkriegszeit, in: Gerhard Baader/Veronika Hofer/Thomas Mayer (Hrsg.), Eugenik in Österreich. Biopolitische Strukturen von 1900-1945, Wien 2007, S. 162-183, hier S. 165 
der treuen Pflegerin." 42 Dabei liege es an einer Kommission, bestehend aus zwei Ärzten und einem Juristen, eine Euthanasie zu beschließen. ${ }^{43}$

Auch bei Alfred Erich Hoche war es die traumatische Erfahrung des Ersten Weltkrieges, in dem Millionen von jungen, „erbgesunden“ Menschen gestorben waren, die ihn zu einem Befürworter der "Vernichtung lebensunwerten Lebens" machte. Hoche unterschied zwei Gruppen von „Blödsinnigen“: Die erste Gruppe umfasste jene PatientInnen, deren geistige Krankheit sich erst im Laufe des Lebens einstellte, so zum Beispiel ältere Menschen oder Menschen mit Schizophrenie. War der "geistige Tod" - wie es Hoche gern nannte - von Geburt an gegeben, gehörten diese Menschen in die zweite Gruppe. Je nach Gruppe müssten dann andere Maßstäbe angewendet werden. Hoche etablierte in seinem Werk mehrere Begriffe, die unter anderem die Nationalsozialisten in ihr Programm dankbar aufnahmen. Er sprach von „Ballastexistenzen“ ,"Menschenhülsen“" "geistig Toten" und "Defektmenschen“. Angesichts derartiger Begriffe ist klar, dass Hoche nicht viel Mitleid für Menschen mit Behinderung hatte, ihnen sogar jegliches Mitleid absprach: „Mitleid ist den geistig Toten gegenüber im Leben und im Sterbensfall die an letzter Stelle angebrachte Gefühlsregung; wo kein Leiden ist, ist auch kein mit-Leiden [sic!]."44 Die Kindereuthanasie und die eugenischen Entwicklungen im Dritten Reich hatte er nach eigenen Angaben später allerdings keineswegs gutgeheißen. ${ }^{45}$

1921 erschien ein zweibändiges Werk, das kurze Zeit später zum Standardwerk der "Rassenhygiene" in Deutschland avancierte: der "Grundriß der menschlichen Erblichkeitslehre und Rassenhygiene". Seine drei Autoren und der Verleger waren allesamt Mitglieder der DGR: Erwin Baur, Arzt, Botaniker und Professor für Vererbungswissenschaft in Berlin, Eugen Fischer, Rassenforscher und Professor für Anatomie in Freiburg, und Fritz Lenz, der mit seiner Habilitationsschrift „Erfahrungen über Erblichkeit und Entartung an Schmetterlingen“ auf sich aufmerksam gemacht hatte. Der Verleger des Werkes, das nach seinen Autoren allgemein der "Baur-Fischer-Lenz" genannt wird, war Julius Friedrich Lehmann, NSDAP-Mitglied seit 1920 und ab 1922 zudem Verleger vom „Archiv für Rassen- und Gesellschafts-Biologie".46

Jeder der Autoren schreibt über eines seiner Spezialgebiete: Fischer berichtet unter anderem über "Die Rassenunterschiede des Menschen“. Baur kommt in seinem Artikel („Abriß der allgemeinen Variations- und Erblichkeitslehre") zum Schluss, dass die "Verhinderung der natürlichen Auslese“ dazu führt, dass "die ständig entstehenden minderwertigen Mutanten nicht ausgemerzt werden“;47 Lenz plädiert für die Tötung „von

42 Karl Binding/Alfred Hoche, Die Freigabe der Vernichtung lebensunwerten Lebens. Ihr Maß und ihre Form, Leipzig 1920, S. 31 f., zit. n. Klee, Euthanasie, S. 23.

43 Ebd., S. $22 \mathrm{f}$.

44 Binding/Hoche, Freigabe, S. 59, zit. n. ebd., S. 26

45 Ebd.

46 Ebd., S. 27.

47 Erwin Baur, Abriß der allgemeinen Variations- und Erblehre, in: Erwin Baur/Eugen Fischer/Fritz Lenz (Hrsg.), Menschliche Erblehre und Rassenhygiene (Eugenik). Band I: Menschliche Erblehre, München 19364, S. 1-94, hier S. 89 . 
Geisteskranken, schweren Psychopathen, Säufern, Schwindsüchtigen, Tauben, Blinden, Zuckerkranken usw".48

Lenz, ab 1923 Lehrstuhlinhaber für "Menschliche Erblehre" in München, hatte für Adolf Hitler stets lobende Worte übrig: „Jedenfalls hat er [Hitler, T. W.] die wesentlichen Gedanken der Rassenhygiene und ihre Bedeutung mit großer geistiger Empfänglichkeit und Energie sich zu eigen gemacht."49 Die Tatsache, dass der spätere Führer höchstpersönlich den „Baur-Fischer-Lenz" gelesen hatte, erfüllte die Autoren und besonders Fritz Lenz mit Stolz. ${ }^{50}$

Der "Baur-Fischer-Lenz", der in den 1920er-Jahren in mehrfachen Auflagen erschien, löste Schallmayers „Vererbung und Auslese“ ab, das bis dahin das Standardwerk der Eugenik in Deutschland war. Ein Vergleich der beiden Werke zeigt eine eindeutige Radikalisierung in den Schlussfolgerungen im „Baur-Fischer-Lenz”. Hatte Schallmayer noch Eheverbote für Erbkranke in den Mittelpunkt gestellt, gingen Baur, Fischer und vor allem Lenz so weit, die Sterilisierung explizit zu fordern. ${ }^{51}$ Hochrechnungen von Grotjahn zufolge müssten laut Lenzschen Kriterien etwa zwanzig Millionen Deutsche - ein Drittel der gesamten deutschen Bevölkerung - zwangssterilisiert werden. Entsprechend groß war seine Genugtuung, dass Hitler „die Sterilisierung nicht nur für extreme Fälle fordert, was für die Gesundung der Rasse ziemlich bedeutungslos sein würde, sondern sie auf den gesamten minderwertigen Teil der Bevölkerung erstreckt wissen will“.52

Nationalsozialistische Rassenideologie ${ }^{53}$

Die NSDAP nahm zahlreiche Elemente der vorangegangenen rassenhygienischen Debatte in ihre Ideologie auf. Der völkische Staat hatte laut Hitler

„die Rasse in den Mittelpunkt des allgemeinen Lebens zu setzen. Er hat für ihre Reinerhaltung zu sorgen. [...] Er muß [sic!] dafür Sorge tragen, daß [sic!] nur wer gesund ist, Kinder zeugt; daß es nur eine Schande gibt: bei eigener Krankheit und eigenen Mängeln dennoch Kinder in die Welt zu setzen, doch eine höchste Ehre: darauf zu verzichten."54

48 Fritz Lenz, Menschliche Auslese und Rassenhygiene, in: Erwin Baur/Eugen Fischer/Fritz Lenz (Hrsg.), Menschliche Erblehre und Rassenhygiene (Eugenik). Band II: Menschliche Auslese und Rassenhygiene, München 19232, S. 186, zit. n. Klee, Euthanasie, S. 28

49 Fritz Lenz, Die Stellung des Nationalsozialismus zur Rassenhygiene, in: Archiv für Rassen- und Gesellschafts-Biologie 25 (1931), S. 300-308, hier S. 302, zit. n. ebd.

50 Ebd.

51 Schmuhl, Rassenhygiene, S. 46.

52 Lenz, Stellung, S. 304, zit. n. ebd., S. 48.

53 Im folgenden Abschnitt wird auf die rassenhygienische und erbbiologische Ideologie der Nationalsozialisten eingegangen; für die gründlichere Darstellung einer umfassenden Rassenideologie (mit Einbezug unter anderem des Antisemitismus und des Rassismus) siehe Johannes Zischka, Die NS-Rassenideologie. Machttaktisches Instrument oder handlungsbestimmendes Ideal? (Geschichte und ihre Hilfswissenschaften 274), Frankfurt am Main-Bern-New York 1986; Thomas Klepsch, Nationalsozialistische Ideologie. Eine Beschreibung ihrer Struktur vor 1933 (Studien zum Nationalsozialismus 2), Münster 1990. 
In der NS-Ideologie waren sowohl die Förderung einer "gesunden Erbmasse" einerseits, als auch die Abwehr von vermeintlich "schädlichem Erbmaterial" andererseits wichtige Grundpfeiler. Dabei war die (vorgeblich) wissenschaftliche Disziplin der "Rassenhygiene" nie ganz deckungsgleich mit dem Nationalsozialismus. Letzterer verband mehrere Strömungen des 19. Jahrhunderts: „Sozialdarwinismus, Pangermanismus, Ariomanie, völkische Ideologie und Rassentheorien".55 Hitler hatte im Grunde zwei Ziele vor Augen, denen er alle anderen ideologischen Prinzipien unterordnete: Zum einen strebte er nach außenpolitischer Macht und Weltherrschaft, zum anderen wollte er die Rassendoktrin in allen Lebensbereichen durchsetzen. ${ }^{56}$ Für Letzteres waren die rassenhygienischen Lehren von Ploetz, Lenz und anderen ebenso wichtig wie die rassistischen und antisemitischen Vorstellungen von Arthur de Gobineau, Houston St. Chamberlain und Paul de Lagarde. Diese beschrieben in ihren Werken sehr ausführlich ihre Ansichten zur Überlegenheit der "germanischen" bzw. "arischen Rasse" und der "Minderwertigkeit" vor allem der "semitischen Rasse".57

Durch die Übernahme derartiger Überlegungen in die Sozial- und Gesundheitspolitik wurde von nun an das Individuum verdrängt, und "das Kollektiv des deutschen Volkes, definiert als erbgesunde, rassisch homogene und leistungsfähige Gemeinschaft",58 in das Blickfeld genommen. Für die Menschen mit Behinderung bedeutete dies, dass sie zu „Ballastexistenzen", "unnützen Essern“59, "Defektmenschen“ oder "leeren Menschenhülsen"60 degradiert wurden. Mit einem derartigen Vokabular zielte das NSRegime darauf ab, die Kosten der Patienten im Vergleich zu deren Nutzen für die „Volksgemeinschaft" in den Vordergrund zu rücken. ${ }^{61}$ Dies war Teil einer Propaganda, die die Bevölkerung von der Notwendigkeit der Sterilisierung (die Ermordungen verliefen ohnehin unter Geheimhaltung) überzeugen sollte. ${ }^{62}$

\section{Euthanasie im Dritten Reich}

\section{Vorläufer und Parallelaktionen der T4}

\section{Gleichschaltung des Gesundheitswesens}

Schon kurz nach der Machtergreifung sicherten sich die Nationalsozialisten die Loyalität der Ärzteschaft, indem sie berufsständische Forderungen erfüllten. Auch Ärz-

\footnotetext{
55 Peter Weingart et al., Rasse, Blut und Gene. Geschichte der Eugenik und Rassenhygiene in Deutschland, Frankfurt am Main 1988, S. 370.

56 Ebd., S. 371.

57 Dazu vertiefend Klepsch, Ideologie, S. 87-98.

58 Brigitte Kepplinger, Zum Gesundheitssystem im Reichsgau Tirol-Vorarlberg. Eine Skizze, in: Bertrand Perz/Thomas Albrich/Elisabeth Dietrich-Daum et al. (Hrsg.), Schlussbericht der Kommission zur Untersuchung der Vorgänge um den Anstaltsfriedhof des Psychiatrischen Krankenhauses in Hall in Tirol in den Jahren 1942 bis 1945 (Veröffentlichungen der Kommission zur Untersuchung der Vorgänge um den Anstaltsfriedhof des Psychiatrischen Krankenhauses in Hall in Tirol in den Jahren 1942 bis 1945), Innsbruck 2014, S. 83-89, hier S. 83.

59 Zit. n. Schreiber, Nationalsozialismus, S. 210.

60 Zit. n. Wolfgang Benz, Geschichte des Dritten Reiches, Bonn 2010, S. 170.

61 Schmuhl, Rassenhygiene, S. 148.

62 Vertiefend zu der NS-Propaganda im Rahmen der rassenhygienischen Politik ebd., S. 173-177.
} 
te, die nicht bereits Mitglieder der NSDAP waren, erwiesen sich - zumindest in der Anfangszeit - als durchaus loyale Anhänger. Als „tiefgreifendste und weitreichendste Gleichschaltungsmaßnahme"63 kann die Neuordnung des Kassenarztwesens angesehen werden. Das Krankenversicherungsgesetz von 1883 hatte die Ärzte in eine Abhängigkeit von den Krankenkassen gebracht, die sie in ihrem Selbstverständnis nicht akzeptieren wollten. Mit Wohlwollen nahmen sie zur Kenntnis, dass die Nationalsozialisten daran waren, „den Saustall der roten Ortskrankenkassen aus[zu]misten", 64 wie es der Reichsminister für Volksaufklärung und Propaganda Joseph Goebbels formulierte. Bis Ende 1933 gelang es den Nationalsozialisten, die Krankenkassenselbstverwaltungen zu zerschlagen. ${ }^{65}$ Mit dem "Gesetz zur Wiederherstellung des Berufsbeamtentums" vom 7. April 1933 konnte die Regierung gleichzeitig nicht-jüdische Ärzte entlassen. Sozialistische und Sozialdemokratische Mediziner wurden ebenfalls ihres Amtes enthoben und verfolgt. ${ }^{6}$ Die bürokratische Gleichschaltung erfolgte mit dem "Gesetz zur Vereinheitlichung des Gesundheitswesens" vom Juli 1934, das sogenannte „Beratungsstellen für Erb- und Rassenpflege" bei den Gesundheitsämtern einrichten ließ. ${ }^{67}$ Deren Aufgabe bestand vor allem darin, (mögliche) „Erbkranke“ dem Amtsarzt zu melden; dieser nahm die Kontrollen vor und legte „Erbarchive“ bzw. „Erbkarteien“ an. ${ }^{68}$ Die Gleichschaltung erfolgte auch auf wissenschaftlicher Seite, indem das Nazi-Regime die Verbreitung von rassenhygienischen Lehren förderte.

Insgesamt lässt sich feststellen, dass kaum eine andere Berufsgruppe derart hinter den Nationalsozialisten stand wie jene der Ärzte. Schätzungen beziffern den Anteil der NSDAP-Mitglieder innerhalb der Ärztekammer auf 45 Prozent, während von den Lehrpersonen beispielsweise „lediglich“ 25 Prozent der Partei beitraten. ${ }^{69}$

\section{Sterilisierungen}

Am 14. Juli 1933, nicht einmal ein halbes Jahr nach der Machtergreifung Hitlers, wurde das "Gesetz zur Verhütung erbkranken Nachwuchses” (GzVeN), kurz „Erbgesundheitsgesetz", verabschiedet. Das Gesetz listet dabei alle Krankheiten und Behinderungen auf, die als sogenannte „Erbkrankheiten“ galten:

„Erbkrank im Sinne dieses Gesetzes ist, wer an einer der folgenden Krankheiten leidet: 1. angeborenem Schwachsinn ${ }^{70}$, 2. Schizophrenie, 3. zirkulärem (manisch-depressivem) Irresein, 4. erblicher Fallsucht, 5. erblichem Veitstanz (Huntingtonsche Chorea), 6. erblicher Blindheit, 7. erblicher Taubheit,

63 Schmuhl, Rassenhygiene, S. 138.

64 Zit. n. ebd., S. 140.

65 Ebd., S. 138-140.

66 Klee, Euthanasie, S. $36 \mathrm{f}$.

67 Elisabeth Dietrich-Daum, Die Psychiatrische Heil- und Pflegeanstalt Hall in Tirol 1830 bis 1940, in: Perz et al. (Hrsg.), Schlussbericht, S. 109.

68 Schmuhl, Rassenhygiene, S. 145.

69 Ebd., S. 37

70 Explizit wurde hier der Terminus „angeboren“ anstatt "erblich“ verwendet, da die Erblichkeit von „Schwachsinn”, d. h. (schwerer) geistiger Behinderung, keineswegs als erwiesen galt. 
8. schwerer erblicher körperlicher Mißbildung [sic!]. Ferner kann unfruchtbar gemacht werden, wer an schwerem Alkoholismus leidet." ${ }^{\prime 1}$

Statistiken zeigen, dass 96 Prozent der sterilisierten Personen aufgrund von "Schwachsinn" (52,9 Prozent), Schizophrenie (25,4 Prozent), Epilepsie (14 Prozent) oder manischen Depressionen (3,2 Prozent) dem Gesetz zum Opfer fielen. Den Rest stellten Alkoholiker (2,4 Prozent), Taube (1 Prozent), Blinde (0,6 Prozent), Körperbehinderte (0,3 Prozent) und Menschen mit Chorea Huntington (0,2 Prozent) dar. ${ }^{72}$ Über eine Zwangssterilisierung entschieden mehr als zweihundert sogenannte Erbgesundheitsgerichte, die im Deutschen Reich eine Art „rassenhygienische Sonderjustiz"73 darstellten, ungeachtet der Tatsache, dass es nach dem wissenschaftlichen Stand der damaligen Zeit in den allermeisten Fällen gar nicht möglich war, Erbkrankheiten festzustellen. ${ }^{74}$

\section{Kindereuthanasie}

Laut einer Legende stand am Anfang der Kindereuthanasie der sogenannte „Fall Knauer" im Jahr 1939. Demnach soll ein Elternpaar der Kanzlei des Führers ein Gesuch gestellt haben, um ihrem körperlich schwerst behinderten und blinden Kind den "Gnadentod" gewähren zu dürfen. ${ }^{75}$ Während einige dieser Geschichte eine große Bedeutung beimessen, ${ }^{76}$ handelt es sich für andere beim "Kind Knauer" um eine von bestimmten Kreisen der Nationalsozialisten hochgeschaukelte Episode, die die Euthanasie an Kindern mit Behinderung rechtfertigte. ${ }^{77}$ Alles in Allem dürfte das "Kind Knauer" nicht der Anlass zur Massentötung von Kindern gewesen sein, doch aber einen wichtigen Vorwand für die beteiligten Personen gespielt haben.

Die Kindereuthanasie unterlag von Beginn an strenger Geheimhaltung. Als zentrale Schaltstelle wurde der Reichsausschuß zur wissenschaftlichen Erfassung von erb- und anlagebedingten schweren Leiden damit beauftragt. Im Hintergrund war aber, zumindest zu Beginn, auch Viktor Brack, Oberdienstleiter des Hauptamtes II der Kanzlei des Führers (KdF), involviert. ${ }^{78}$

Im August 1939 wurden mittels eines streng geheimen Erlasses des Reichsministerium des Innern (RMdl) alle Hebammen, Ärzte und sonstigen Geburtshelfern darauf angewiesen, Kinder, die bei der Geburt bereits Behinderungen aufwiesen, zu melden. ${ }^{79}$ Die Operation ging zu Beginn nur schleppend voran. Erst allmählich steigerte sich die Bereitschaft der Ärzte, der Aufforderung nach einer Meldung nachzukommen. Die Mel-

71 Gesetz zur Verhütung erbkranken Nachwuchses, § 1, zit. n. Klee, Euthanasie, S. 39; Schmuhl, Rassenhygiene, S. 156.

72 Zahlen aus Wolfgang Form, Das Gesetz zur Verhütung erbkranken Nachwuchses und seine Entwicklung nach 1945 in Hessen, in: Gerhard Aumüller (Hrsg.), Kontinuität und Neuanfang in der Hochschulmedizin nach 1945, Marburg-Schüren 1997, zit. n. Klee, Euthanasie, S. 40 f.; Schmuhl, Rassenhygiene, S. 156.

73 Klee, Euthanasie, S. 42.

74 Schreiber, Nationalsozialismus, S. 211.

75 Klee, Euthanasie, S. 81-83.

76 So zum Beispiel Schmuhl, vgl. Schmuhl, Rassenhygiene, S. 182.

77 Diese Ansicht teilt Klee: Klee, Euthanasie, S. 81-83.

78 Schmuhl, Rassenhygiene, S. 182.

79 Wolfgang Neugebauer, NS-Euthanasieaktionen in Österreich. Ein Überblick, in: Perz et al. (Hrsg.), Schlussbericht, S. 37. 
debögen wurden in der KdF von Beamten und später von drei medizinischen Gutachtern durchgesehen, die mittels Plus- und Minuszeichen direkt auf dem Bogen über das Leben der/s Neugeborenen entschieden. ${ }^{80}$ Die Tötungen fanden zu einem großen Teil in den oftmals neu eingerichteten sogenannten „Kinderfachabteilungen" (insgesamt dreißig im gesamten Deutschen Reich ${ }^{81}$ ) in den Krankenhäusern statt. Viele Kinder wurden in Gaswagen ermordet - besonders in der Frühphase. Der Großteil der in Frage kommenden Buben und Mädchen wurde mittels Luminal ${ }^{82}$ und anderer Schlafmittel getötet. Den Eltern wurde vorgegaukelt, man würde bei den Kindern neue, oftmals riskante Therapien anwenden. Die „Therapien“ überlebten die Kinder nie. ${ }^{83}$

\section{Die "Aktion T4"}

Beginn und Apparat

"Reichsleiter Bouhler und Dr. med. Brandt sind unter Verantwortung beauftragt, die Befugnisse namentlich zu bestimmender Ärzte so zu erweitern, daß nach menschlichem Ermessen unheilbar Kranken bei kritischster Beurteilung ihres Krankheitszustandes der Gnadentod gewährt werden kann. gez.: Adolf Hitler" ${ }^{\prime \prime 4}$

Mit diesen Worten gab Hitler den Auftrag zu den Euthanasiemorden, der in den folgenden zwei Jahren laut Hartheimer Statistik 70.273 Menschen das Leben kostete. ${ }^{85}$ Obwohl er den Befehl im Oktober 1939 weiterleitete, datierte Hitler ihn auf den 1. September, dem Tag des Beginns des Krieges. ${ }^{86}$ Die in dem Erlass genannten Personen waren der Leiter der Kanzlei des Führers Reichsleiter Philipp Bouhler und sein Leibarzt Prof. Dr. Karl Brandt. Die Aktion stand von Beginn an unter strenger Geheimhaltung. Allen Beteiligten war klar, dass sie in einer rechtlichen Grauzone agierten, und die „Aktion“ im strafrechtlichen Sinne als Mord einzustufen war. ${ }^{87}$

Bouhler, Brandt und Ministerialdirigent Dr. Herbert Linden vom Reichsministerium des Innern bauten schon Monate vor dem Führererlass eine geheime Organisation auf. Durch den Einbezug von Linden wollte man sich der Unterstützung des RMdl, ohne die eine planmäßige Erfassung der PatientInnen nicht möglich gewesen wäre, sicher sein. ${ }^{88}$ Das Zentrum der "Aktion“ war das Hauptamt II der Kanzlei des Führers, dessen Leitung Viktor Brack innehatte. Damit unterstand dieser direkt Bouhler und Brandt. Dem Hauptamt II untergeordnet war die Zentraldienststelle in Berlin mit Adresse Tier-

80 Schmuhl, Rassenhygiene, S. 184.

81 Neugebauer, NS-Euthanasieaktionen, S. 37.

82 Luminal, eigentlich Phenobarbital, ist ein Schlafmittel und wurde bis weit in die zweite Hälfte des 20. Jahrhunderts hinein gegen Epilepsie und andere Krankheiten verwendet.

83 Klee, Euthanasie, S. 335-343.

84 Geheimer Führererlass, datiert auf den 1. September 1939, zit. n. Schmuhl, Rassenhygiene, S. 190.

85 Andrea Kammerhofer, „Bis zum 1. September 1941 wurden desinfiziert: Personen: 70.273“. Die „Hartheimer Statistik", in: Kepplinger et al. (Hrsg.), Tötungsanstalt Hartheim, S. 117-130, hier S. 124

86 Neugebauer, "Aktion T4", S. 19.

87 Ebd

88 Brigitte Kepplinger, NS-Euthanasie in Österreich. Die „Aktion T4” - Struktur und Ablauf, in: Kepplinger et al. (Hrsg.), Tötungsanstalt Hartheim, S. 37. 
gartenstraße 4 - oder eben „T4".89 Der Leiter davon war Dietrich Allers. Die medizinische Abteilung, der zuerst Prof. Dr. Werner Heyde und ab 1941 Prof. Dr. Hermann Paul Nitsche vorstanden, bildete zusammen mit der Büroabteilung von Gerhard Bohne die Reichsarbeitsgemeinschaft Heil- und Pflegeanstalten (RAG). Die Gemeinnützige Krankentransport GmbH (Gekrat) unter Reinhold Vorberg sollte die Krankentransporte organisieren..$^{90}$ Diese und weitere Scheinunternehmen - mit insgesamt an die etwa fünfhundert MitarbeiterInnen (darunter mindestens fünfzig Ärzte bis Ende der "Aktion"91) - dienten dazu, die zentrale Rolle der KdF bzw. des Hauptamtes II unter Viktor Brack in der gesamten „Aktion" so gut als möglich zu vertuschen. Es handelte sich somit „um Abteilungen ein und derselben Gesamtorganisation" ${ }^{\prime 2}$.

\section{Die Durchführung - von der Erfassung bis zur Tötung}

Der "Offizielle" Beginn der "Aktion T4" im Deutschen Reich kann mit 9. Oktober 1939 datiert werden: An diesem Tag schickte das RMdl einen Runderlass an alle Heil- und Pflegeanstalten. Dem Erlass beigefügt waren zwei Meldebögen und ein Merkblatt. Die Anstalten wurden angewiesen - ohne weitere Erklärungen -, psychisch kranke und/ oder behinderte PatientInnen zu melden. Diese wurden im ersten Bogen eingetragen, während im zweiten Informationen zur Anstalt (Größe, Betten, Personal, Anschluss an Bahnschienen, nächster Bahnhof etc.) erfasst wurden. Ähnlich wie bei der Durchführung der Kindereuthanasie wurden die - anfangs nur sehr zögerlich - zurückgesandten Bögen von drei "Gutachterlnnen" der medizinischen Abteilung der Zentraldienststelle mit Plus- und Minuszeichen versehen. ${ }^{93}$ Dabei entschieden die "Gutachterlnnen" unter anderem anhand der Angaben zur Arbeitsfähigkeit und eventuell zur Art der Beschäftigung der einzelnen AnstaltsinsassInnen. Die Bögen wurden dann noch einem der sogenannten „Obergutachtern“ (Linden, später Heyde und Nitsche) vorgelegt, die eine endgültige Entscheidung trafen. Die ausgewählten Bögen wurden darauf der Gekrat übergeben, die die Transporte in die Euthanasieanstalten organisierte. ${ }^{94}$

So waren für die mindestens 100.000 PatientInnen an die vierzig „Begutachter“ zuständig. Über 70.000 Menschen wurden dann schließlich den sechs Tötungsanstalten übergeben, wo sie einen grausamen Tod fanden. In den Gaskammern der Anstalten wurden die ohnehin schon sehr geschwächten Ankömmlinge durch Kohlenmonoxid erstickt. Die sechs Tötungsanstalten waren: Grafeneck/Württemberg (9.839 „Desinfizierungen“), Brandenburg a. d. Havel (9.772), Hartheim (18.269), Sonnenstein/Pirna (13.720), Bernburg a. d. Saale (8.601), Hadamar/Limburg (10.072). ${ }^{95}$

89 Der Umzug in eine Villa in der Tiergartenstraße 4 erfolgt erst im April 1940, vgl. Klee, Euthanasie, S. 121.

90 Neugebauer, "Aktion T4", S. 20 f.

91 Schmuhl, Rassenhygiene, S. 192.

92 Aussage Therese Hergenröder, Sekretärin des hessischen Landesrats Bernotat, vom 23.8.1946 im Eichberg-Verfahren, zit. n. Klee, Euthanasie, S. 121. Eine detaillierte Darstellung der internen Struktur und Organisation des T4-Apparats findet sich in ebd., S. 121 f. und in Kepplinger, NS-Euthanasie, S. 39 und in Neugebauer, NS-Euthanasieaktionen, S. 39.

93 Neugebauer, "Aktion T4", S. 22 f.

94 Ebd., S. 24

95 Ebd. 
Alle bei der "Aktion T4" beteiligten Personen und Institutionen übten sich in Geheimhaltung. Die Morde wurden mittels der Angabe von natürlichen Todesursachen vertuscht. Auch der Todesort und -tag wurden falsch angegeben. Damit sollte die Tatsache verschleiert werden, dass es sich um Massentötungen in immer denselben Anstalten handelte. Die Briefe, die die traurige Nachricht vom Tod einer/s Insassin/Insassen der Familie mitteilen sollten, wurden nach stets gleichen Mustern verfasst. Die Leichen wurden mit dem Hinweis auf eine "seuchenpolizeiliche Anordnung" verbrannt, den Verwandten eine Urne mit einer $x$-beliebigen Asche gesendet. ${ }^{96}$

\section{Widerstand}

Trotz dieser Vorsichtsmaßnahme kamen in der Bevölkerung immer wieder Gerüchte auf, dass die verstorbenen Verwandten in den Anstalten ermordet wurden. Immer wieder soll es zu Ansammlungen von Menschen vor den Anstalten gekommen sein, als die Gekrat mit ihren Bussen eintraf. ${ }^{97}$ Auch das Ausland wurde auf das Euthanasieprogramm aufmerksam. In einer von der Royal Air Force über deutsche Städte abgeworfenen Propagandazeitung wurde beispielsweise auf den Wiener Arzt und „T4"-Beteiligten Dr. Erwin Jekelius aufmerksam gemacht. Auch innerhalb der AnstaltspatientInnen verbreitete sich das Gerücht der Tötungen. Die Angehörigen weigerten sich in der Folge immer mehr, hilfsbedürftige Verwandte an Anstalten abzugeben. ${ }^{98}$

Der lauteste Widerstand kam vom kirchlich-katholischen Lager. Besonderen Ruhm in diesem Zusammenhang erlangte der Bischof von Münster Clemens August Graf von Galen, der in diversen Predigten und Schriften die Euthanasie ansprach und verurteilte. ${ }^{99}$ Sein offenes Ansprechen der Tötungen ermutigte andere Bischöfe und Pfarrer dazu, in Reden darauf einzugehen - eine Tatsache, die im totalitären System des nationalsozialistischen Staates als bemerkenswert angesehen werden kann. ${ }^{100}$

"Sonderbehandlung 14f13" - die Ermordung von KZ-Häftlingen in den Euthanasieanstalten

„T4"-Gutachter wurden ab Anfang 1941 auch von den Leitungen der Konzentrationslager "ausgeliehen", wo sie "Schwerstkranke" - Häftlinge, die als arbeitsunfähig eingestuft worden waren - im Rahmen der "Aktion T4" ermordeten. „14" stellte das Kürzel für Todesfälle in den KZ dar, "13" stand für den Tod durch Vergasen. Die von den Gutachtern ausgewählten Personen wurden wiederum durch die Gekrat in eine der Tötungsanstalten gebracht und dort vergast. ${ }^{101}$ Wie die ärztlichen Gutachter der "Aktion T4" die "Sonderbehandlung 14f13" wahrnahmen, kann zum Teil aus dem sehr umfangreichen Briefverkehr von Dr. Friedrich Mennecke, der bereits zuvor unter anderem die Heil-

\footnotetext{
96 Schmuhl, Rassenhygiene, S. 208.

97 Ebd., S. 209.

98 Neugebauer, "Aktion T4", S. 28 f.

99 Ebd., S. 29; Klee, Euthanasie, S. 255 f.

100 Ebd., S. $258 \mathrm{f}$.

101 Schmuhl, Rassenhygiene, S. $217 \mathrm{f}$.
} 
und Pflegeanstalt in Hall in Tirol gesichtet hatte, ${ }^{102}$ abgelesen werden: „Auf geht's zum fröhlichen Jagen!!! Ich bin frisch $u$. munter, hoffentlich Du auch, Herzli!"103 Kepplinger und Reese schätzen die Zahl der durch die "Sonderbehandlung 14f13" ermordeten KZInsassInnen auf 10.000 bis 20.000, ${ }^{104}$ Schmuhl auf 20.000. ${ }^{105}$ "14f13" stellte damit eine Art Bindeglied zwischen dem Apparat der Euthanasiemorde und jenem der Konzentrationslager dar. ${ }^{106}$

Das Ende von „T4" und die Weiterführung der Morde als "dezentrale Anstaltsmorde"107

Am 24. August 1941 erteilte Hitler direkt an Brandt den Befehl, die "Aktion T4" mit einem Schlag zu beenden. ${ }^{108}$ Die Überraschung darüber in den einzelnen Anstaltsleitungen, die telefonisch darüber benachrichtigt worden waren, war groß. Die Ursachen für das offenbar plötzliche Umdenken Hitlers sind nicht ganz klar. Fakt ist aber, dass die Unruhe innerhalb der Bevölkerung in einem für den Führer unannehmbaren Ausmaß zugenommen hatte: Es war der Zeitpunkt des Kriegsbeginns mit der Sowjetunion, für dessen Umsetzung der Führer den Rückhalt in „seinem Volk" benötigte. ${ }^{109}$ Besonders die Predigt des Bischofs von Münster, von Galen, vom 3. August 1941 spielte eine wichtige Rolle in der Aufklärung der Bevölkerung. In der Folge wuchs der Unmut über die dargestellten Ereignisse, und das NS-Regime sah sich unter Druck gesetzt. ${ }^{110}$ Gleichzeitig war nach der Tötung von über 70.000 PatientInnen das Kurzziel der Freilegung von Betten wohl erfüllt, was den Entschluss zur Beendigung der "Aktion" erleichtert haben dürfte.

Das Ende der "Aktion T4" bedeutete aber nicht zwangsläufig das Ende des gesamten "T4"-Apparates. So wurde die Kindereuthanasie bis kurz vor Ende des Krieges weitergeführt, ebenso die „Sonderbehandlung 14f13". Gleichzeitig wurden die Heil- und Pflegeanstalten angehalten, die Euthanasie dezentral, sprich vor Ort, weiter durchzuführen. ${ }^{11}$ Durch Überdosierungen von Medikamenten sollten „bereitwillige“ Ärzte die Morde im Sinne einer rassenhygienischen Auslese fortsetzen. Den AnstaltsinsassInnen sollte dreimal täglich Luminal in das Essen gemischt werden; zusammen mit einer radikalen Unterversorgung wirkte das Schlafmittel tödlich. Die Opfer erlitten auf diese Weise einen oft Tage andauernden, qualvollen Tod. ${ }^{112}$ Insgesamt fanden in dieser Phase ungefähr gleich viele Menschen den Tod wie in den beiden Jahren der "Aktion T4".113

102 Schreiber, Nationalsozialismus, S. 218.

103 Dr. Friedrich Mennecke, T4-Arzt, in einem Brief vom 28.11.1941, 7 Uhr 40, an seine Gemahlin, kurz bevor er im KZ Buchenwald in Frage kommende InsassInnen für die Gaskammer auswählte, zit. n. Klee, Euthanasie, S. 284.

104 Brigitte Kepplinger/Hartmut Reese, Die Nationalsozialistische Euthanasieverbrechen. Orte und „Aktionen“, in: Kepplinger et al. (Hrsg.), Tötungsanstalt Hartheim, S. 442.

105 Schmuhl, Rassenhygiene, S. 219.

106 Kepplinger/Reese, Euthanasieverbrechen, S. 443.

107 Für die Verwendung der Begrifflichkeiten wilde Euthanasie bzw. dezentrale/regionale/regionalisierte Anstaltsmorde siehe Neugebauer, NS-Euthanasieaktionen, S. 43.

108 Schmuhl, Rassenhygiene, S. 210.

109 Kepplinger, NS-Euthanasie, S. 58.

110 Schmuhl, Rassenhygiene, S. $210 \mathrm{f}$.

111 Neugebauer, „Aktion T4", S. 30

112 Schmuhl, Rassenhygiene, S. $220 \mathrm{f}$.

113 Neugebauer, NS-Euthanasieaktionen, S. 43. 


\section{„....alles was unheilbar ist solle weg..."114 - Die Umsetzung der „Aktion T4“ im Reichsgau Tirol-Vorarlberg}

Vor der "Aktion"

Das "Erbgesundheitsgesetz" trat in Österreich mit 1. Januar 1940 in Kraft. Damit begannen die Zwangssterilisationen auch in der neu dazugekommenen „Ostmark”. Horst Schreiber schätzt die Zahl der Personen im Gau Tirol-Vorarlberg, die dieser grausamen Maßnahme zum Opfer fielen, auf rund vierhundert. ${ }^{115}$ Die Existenz von „Kinderfachabteilungen“ für die Ermordung „erbkranker" Kinder im Reichsgau ist nicht belegt. Ob Säuglinge in Tirol und Vorarlberg erfasst und getötet wurden, liegt noch im Unklaren. ${ }^{116}$

Für die Vorbereitungen der "Aktion T4“ im Gau Tirol-Vorarlberg reiste Viktor Brack höchstpersönlich nach Innsbruck. Dort traf er sich mit Gauleiter Franz Hofer um erste Vorkehrungen zu treffen. Auch der Leiter der staatlichen Gesundheitsverwaltung im Gau, Dr. Hans Czermak, und der Gauamtsleiter für Volksgesundheit, Dr. Josef Malfatti, wurden bereits zu diesem Zeitpunkt über die „Aktion“ informiert. In der Zeit nach dem Treffen legten die beteiligten, allen voran Czermak, ein großes Maß an Eigeninitiative an den Tag. ${ }^{117}$ Ende August 1940 traf eine ärztliche Kommission unter Leitung von Dr. Friedrich Mennecke in Hall ein. Diese sah sich die Krankenakten der PatientInnen durch. Auf Grundlage dieser Sichtung - und ohne die betreffenden InsassInnen je zu Gesicht bekommen zu haben - stellte die Kommission später von Berlin aus die Transportlisten zusammen. Czermak schloss in der Zwischenzeit eine Abmachung mit Dr. Rudolf Lonauer, der in Personalunion Anstaltsleiter der Heil- und Pflegeanstalt Niedernhart (Linz), ärztlicher Leiter der Tötungsanstalt Hartheim, Beauftragter der RAG und „T4"-Gutachter war: Es wurde beschlossen, dass die RAG für alle Kosten und das weitere Schicksal der PatientInnen ab der Ankunft der Transporte in Niedernhart zuständig war. ${ }^{118}$ Am 3. Dezember 1940 erhielt Czermak einen Brief aus der Anstalt Niedernhart. In diesem stand, dass eine „Verlegung von Patienten" in den kommenden neun Tagen durchgeführt werden soll. In den folgenden Jahren war es nicht selten die Direktion der Gau-Heil- und Pflegeanstalt Niedernhart in Linz - und im Speziellen der Anstaltsleiter Lonauer selbst -, der Dr. Ernst Klebelsberg von der Anstalt Hall entsprechende Befehle erteilte. ${ }^{119}$

114 Zeugenaussage Dr. Klebelsberg bei der Hauptverhandlung gegen Dr. Czermak vor dem Landesgericht (LG) Innsbruck, 30.11.1949. Tiroler Landesarchiv (TLA), LG Innsbruck, 10 Vr 4740/47, zit. n. Oliver Seifert, Das Sterben in der Heil- und Pflegeanstalt Hall 1942-1945, in: Perz et al. (Hrsg.), Schlussbericht, S. 115.

115 Schreiber, Nationalsozialismus, S. 213.

116 Oliver Seifert, „Sterben hätten sie auch hier können“. Die „Euthanasie“-Transporte aus der Heil- und Pflegeanstalt Hall in Tirol nach Hartheim und Niedernhart, in: Kepplinger et al. (Hrsg.), Tötungsanstalt Hartheim, S. 363.

117 Ebd., S. 364.

118 Ebd., S. 369.

119 Ebd., S. 371. 


\section{Durchführung}

Der erste Transport am 10. Dezember 1940

Im Vorfeld des ersten Transportes reiste Dr. Georg Renno, Stellvertreter von Lonauer in Hartheim, nach Hall, da "etwas nicht ganz in Ordnung [gewesen] sei","20 denn Klebelsberg wollte die Transportlisten nicht ohne Weiteres akzeptieren. Von den insgesamt 291 aufgeführten Personen ${ }^{121}$ waren viele, die laut dem Haller Primar noch arbeitsfähig und die deshalb für die Anstalt von Wert waren. Zusammen mit Dr. Helmut Scharfetter, dem Vorstand der Psychiatrischen Klinik Innsbruck, NSDAP-, NS-Ärztebund- und SS-Mitglied, unterbreitete er seine Sorge Czermak, der das Problem Gauleiter Hofer vortrug. Czermak erhielt für Klebelsberg eine Ermächtigung, alle heilbaren PatientInnen von der Liste streichen zu dürfen. ${ }^{122}$ Mit dieser "Generalvollmacht"123 ausgestattet, konnte Primar Klebelsberg insgesamt 112 Menschen vor der Fahrt nach Hartheim - und damit dem sicheren Tod - bewahren. Damit konnte Klebelsberg eine begrenzte Anzahl an Leben retten, hatte aber auch eine ambivalente Rolle inne: Falls er zu viele Menschen von der Liste strich, riskierte er nach eigenen Aussagen durch einen linientreuen NS-Arzt ersetzt zu werden. ${ }^{124}$ Seine Rolle relativierte Klebelsberg im Nachhinein mit den Worten: „Ich habe die Auswahl getroffen, ob der wegkommt oder nicht, aber nicht darüber, ob er leben dürfe oder nicht. Wenn ich die Zustimmung nicht gegeben hätte, wären die Leute doch nicht gerettet worden." ${ }^{25}$ Die Auswahl traf er anhand der Arbeitsleistung der PatientInnen.

Die 111 Männer und 68 Frauen, die nicht von der Liste gestrichen wurden, wurden am 10. Dezember 1940 von Einheiten Rennos mit Autobussen zum Bahnhof in Hall gebracht und von dort mit dem Zug direkt nach Hartheim „verlegt“. Zuvor war ihnen noch eine Marke um den Hals gehängt und ihr Besitz mitgegeben worden. ${ }^{126}$

Neben diesem Transport aus Hall kam es zu einem weiteren aus dem St. Josefs-Institut in Mils, bei dem von ursprünglich 122 geforderten Personen 67 schlussendlich wegtransportiert wurden - wiederum erfolgte eine Streichung mit dem Verweis auf die Arbeitsleistung der Betroffenen. Zudem konnte ein Mann kurz vor der Abfahrt fliehen. ${ }^{27}$

120 Staatsanwaltschaft (StA) Konstanz, 2 Js 524/61, Zeugenaussage Dr. Georg Renno vor der Polizei Frankenthal/Pfalz, 19.2.1963, zit. n. ebd., S. 372.

121 Seifert, Heil- und Pflegeanstalt, S. 113.

122 Friedrich Stepanek, Zur Untersuchung des Personals der Heil- und Pflegeanstalt Hall, in: Perz et al. (Hrsg.), Schlussbericht, S. $189 \mathrm{f}$.

123 Seifert, Sterben, S. 374.

124 Archiv Edwin Tangl (Privatbesitz Rainer Hofmann), Bericht über die Vernehmung von Dr. Klebelsberg durch die Bundespolizeidirektion Innsbruck, 15.5.1946, zit. n. ebd., S. 375.

125 Zit. n. Horst Schreiber, Ein „Idealist, aber kein Fanatiker“? Dr. Hans Czermak und die NS-Euthanasie in Tirol, 2008, [http://www.horstschreiber.at/texte/czermak-und-die-nseuthanasie-in-tirol], eingesehen 19.9.2014.

126 Seifert, Sterben, S. 378.

127 Ebd., S. 379. 
Der zweite Transport am 20. März 1941

Anfang des Jahres 1941 war es für Czermak sowie Klebelsberg klar, dass es zu weiteren Deportationen kommen würde. Im Vorfeld dieses Transportes wurden auch die Versorgungs-, Armen- und Altenheime von Czermak, Lonauer und Renno besichtigt. Am 14. März folgte der Abtransport von zwanzig Personen aus dem Versorgungshaus Nassereith sowie 19 Patientinnen aus dem Versorgungshaus Imst. Mit Bussen wurden sie nach Hall transferiert. Die Zusammenführung von PatientInnen in der HPA Hall in Tirol diente wohl dem reibungslosen Ablauf der "Aktion“ in Tirol. ${ }^{128}$

Auch aus der Heil- und Pflegeanstalt Valduna bei Rankweil (Vorarlberg) wurden drei Transporte nach Hall getätigt, zwei Transporte wurden am 10. Februar und am 17. März 1941 von Valduna direkt nach Hartheim zur Vergasung geschickt. ${ }^{129}$ Laut Hinterhuber (auf Erhebungen der Staatsanwaltschaft Konstanz basierend) sollen mindestens 447 PatientInnen aus Vorarlberg „verlegt“ worden sein. 220 davon wurden direkt nach Hartheim gebracht. ${ }^{130}$ Valduna wurde mehr oder weniger "geleert" und von der Wehrmacht als Lazarett verwendet.

Schließlich wurden am 20. März aus Hall 92 Menschen abtransportiert. Dabei strich dieses Mal Klebelsberg nur mehr drei Personen von den Listen. Dies hing damit zusammen, dass er bereits im Vorfeld Halbjahresberichte erstellt hatte, in denen er auf die Arbeitsfähigkeit von einigen der InsassInnen verwiesen hatte. Auf den Transportlisten, die sich nach den Halbjahresberichten richteten, waren demnach nur sehr wenige Personen, die Klebelsberg mit dem Argument der Arbeitsfähigkeit in Hall behalten konnte. ${ }^{131}$ Bei den Verhandlungen des Volksgerichtsprozesses vom Jahr 1949 gegen Czermak rechtfertigte Klebelsberg seine Rolle: „Beim zweiten Transport ist nur weggegangen, was ich verantworten konnte. Renno hat mir Richtlinien gegeben in dem Sinne, alles was unheilbar ist solle weg."132

Der dritte Transport am 29. Mai 1941

Die Leitung der Anstalt Hartheim kündigte bis Mitte Mai einen erneuten Transport an. In der Zwischenzeit forcierten Czermak und Lonauer die Konzentration von geistig behinderten Menschen in der HPA Hall in Tirol, während die Armen- und Versorgungshäuser alten und körperlich behinderten Personen vorbehalten werden sollten. Über den dritten Transport ist nur wenig bekannt. Man weiß nur von 29 Personen sicher, dass sie am 29. Mai 1941 von Hall wegtransportiert worden sind: „21 Frauen aus dem

128 Seifert, Sterben, S. 379-381.

129 Ebd., S. 381.

130 Hartmann Hinterhuber, Ermordet und vergessen. Nationalsozialistische Verbrechen an psychisch Kranken und Behinderten in Nord- und Südtirol, Innsbruck 1995, S. 47.

131 Seifert, Sterben, S. 383.

132 Zeugenaussage Dr. Klebelsberg bei der Hauptverhandlung gegen Dr. Czermak vor dem Landesgericht (LG) Innsbruck, 30.11.1949. Tiroler Landesarchiv (TLA), LG Innsbruck, 10 Vr 4740/47, zit. n. Seifert, Heil- und Pflegeanstalt, S. 115 . 
Versorgungshaus Ried [...], vier Männer und zwei Frauen aus der Anstalt Hall [...] und je ein Mann und eine Frau aus dem St. Josefs-Institut in Mils [....."133

Interessant ist die Intervention des Innsbrucker Oberbürgermeisters Egon Denz bei Gauleiter Hofer, um einen Abtransport aus den Innsbrucker Heimen zu verhindern. Dies beweist, dass Einflussnahmen im kleinen Rahmen durchaus möglich waren. ${ }^{134}$

Der vierte Transport am 31. August 1942

Im August 1941 wurde die "Aktion T4" von Berlin aus eingestellt. Umso erstaunlicher ist es, dass es rund ein Jahr später zu einem erneuten Transport aus Hall, diesmal nach Niedernhart, kam. Nicht nur aus diesem Grund war dieser vierte und letzte Transport aus dem Gau Tirol-Vorarlberg einzigartig:135 Auch die Durchführung der Morde mittels einer Überdosierung von Arzneimitteln war in diesem Zusammenhang eine Neuheit. ${ }^{136}$

Dem Transport am 31. August 1942 gingen energische Interventionen von Czermak bei Lonauer und bei zuständigen Stellen in Berlin voraus. Er klagte über die „überfüllte” Anstalt Hall in Tirol, was laut ihm „ein ganz außerordentlich beklagenswerter Zustand"137 war. Schlussendlich erreichte Czermak den gewünschten Abtransport. Oliver Seifert nimmt an, dass die Transportlisten aus einem Zusammenwirken vor allem von Czermak und Lonauer, aber in begrenzten Umfang auch Klebelsberg, entstanden waren. ${ }^{138}$ Mit zwei Bussen wurden die 60 PatientInnen diesmal von den eigenen Pflegerlnnen der HPA Hall in Tirol bis nach Niedernhart gebracht. Der dortige Anstaltsleiter Lonauer hatte bereits im Vorfeld eine Art „Tötungsstation“ eingerichtet, in der alle sechzig Ankömmlinge "zu Tode gespritzt"139 wurden.

Später benachrichtigte Lonauer Czermak schriftlich über den Tod der PatientInnen. Dabei bemerkte er, dass „diese Behandlungsmethode praktischer und reibungsloser ist als die frühere". 140

\section{Reaktionen der Opfer}

Reaktionen von InsassInnen, die von Tirol aus nach Hartheim bzw. Niedernhart gebracht wurden, sind sehr spärlich erhalten. Es ist davon auszugehen, dass viele der Opfer - vor allem jene, die nur leichte Behinderungen hatten - von ihrem Schicksal wussten bzw. es zumindest erahnten. Es sind hierzu nur einige wenige Zeugenaussagen vorhanden, wie zum Beispiel jene der Schwester Oberin, einer Pflegerin von Nassereith:

133 Seifert, Sterben, S. 386.

134 Ebd.

135 Ders., Heil- und Pflegeanstalt, S. 115.

136 Ders, Sterben, S. 401.

137 TLA, Reichsstatthalterei (RStH) in Tirol und Vorarlberg, Abt. IIla1 (medizinische Angelegenheiten), ZI. M-XI 1941 (Heil- und Pflegeanstalten), Dr. Czermak an Dr. Lonauer, 3.7.1942, zit. n. ebd., S. 398.

138 Ebd., S. 399; Seifert, Heil- und Pflegeanstalt, S. 115.

139 Seifert, Sterben, S. 401.

140 TLA, RStH in Tirol und Vorarlberg, Abt. Illa1 (medizinische Angelegenheiten), ZI. M-XI 1941 (Heil- und Pflegeanstalten), Dr. Lonauer an Dr. Czermak, 5.11.1942, zit. n. ebd., S. 402. 
„Die Pfleglinge machten keine besonderen Schwierigkeiten, da sie glaubten, es handle sich um eine Spazierfahrt. Nur ein Pflegling aus Imst, die sich bereits im Auto befand, schrie wiederholt,,wir kommen unter die Metzger', sodass sogar die Bevölkerung aufmerksam wurde."141

Für die Kinderanstalt Mariathal in Kramsach berichtete Schwester Alberta Berchtenbreiter von 23. Mai 1941, als 61 Kinder mit Bussen abgeholt wurden:

"[...] Im Verbringen in die Autos spielte sich mancher Kampf ab. Ein ganz braver Bub mit 6 Jahren wurde von der Tante abgeholt: Beim Ausgang wurde ihr der Kleine weggerissen und ins Auto verbracht. Ein 14-jähriger Bub verkroch sich im Dach, wo die Männer nicht hin konnten. Alle Kunst, ihn heraufzubringen, war vergeblich. Eine Schwester mußte [sic!] den Versuch machen: Als er die Schwester sah, kam er von selbst herauf - um das Los der anderen zu teilen. Ein 16-jähriger Bub lag schwer krank im Bett. Ich bat den Kleinen dazulassen, er sterbe ja ohnehin gleich. Es war umsonst. Er mußte [sic!] zu den anderen ins Auto [...] Unter den Entführten waren einige größere, ältere Buben und Mädchen, die willig und tüchtig Haus- und Gartenarbeiten verrichteten. Auf die Bitte, diese dazulassen, versprach man, sie wiederzubringen: Es kam aber niemand mehr zurück!"142

Und weiter:

„[...] und alle Kinder, es waren damals 60 [sic!] an der Zahl wurden mit Gewalt und gegen unseren Einspruch weggebracht. Die Kinder haben geweint und gejammert und als sie in den Omnibussen untergebracht worden waren, hörte man die Kinder nicht mehr, sondern wurde es alsbald still. Ich nehme an, bzw. hat es uns damals den Anschein erweckt, dass den Kindern damals irgendwelche Einspritzungen verabreicht wurden."143

Beim Abtransport von InsassInnen des St. Josefs-Institut in Mils am 10. Dezember 1940 konnte ein Mann fliehen; wie viel er von seinem bevorstehenden Schicksal wusste, ist nicht bekannt. ${ }^{144}$ Aus der HPA Hall ist ein Fall überliefert, in dem eine Patientin in grauer Vorahnung einen Brief an ihren Cousin schreibt, er möge sie herausholen, bevor sie zur "Verurnung" gebracht werde. In der Tat war sie beim ersten Transport Ende 1940 dabei. Ihr Cousin erhielt nur mehr eine Urne. ${ }^{145}$

141 TLA, LG Innsbruck, 10 Vr 4740/47 (Verfahren Dr. Hans Czermak), Zeugenvernehmung Sr. Martha Puschmann vor dem LG Innsbruck, zit. n. Seifert, Sterben, S. 389.

142 Zit. n. Hinterhuber, Ermordet, S. 105 f.

143 Archiv Edwin Tang (Privatbesitz Rainer Hofmann), Bericht über die Vernehmung der Leiterin der Idiotenanstalt Mariathal, Alberta Berchtenbreiter, von der Bundespolizeidirektion Innsbruck, 7.12.1946, zit. n. Seifert, Sterben, S. 389 .

144 Ebd., S. 390.

145 Ebd. 


\section{Reaktionen der Verwandten}

Wie im gesamten Deutschen Reich erhielten auch die Verwandten von PatientInnen aus den Tiroler und Vorarlberger Anstalten oft erst Wochen nach dem Tod eine Nachricht mit erfundener Todesursache und Todesort. Dass die zugesandte Urne nicht die Asche der/s Verwandten enthielt, hatte sich auch hier schnell herumgesprochen. Interventionen von Angehörigen direkt bei der Anstaltsleitung in Hall in Tirol bzw. Hartheim hatten nur selten Erfolg. Entweder wurde ihnen der Besuch der - nicht selten bereits ermordeten - PatientInnen verweigert, oder ihnen mitgeteilt, diese seien wiederum in eine andere Anstalt wegtransportiert worden. Auch persönliche Anfeindungen gegen Primar Klebelsberg von Seiten der Angehörigen waren nicht selten. Ihm wurde unter anderem vorgeworfen, über die Köpfe der Angehörigen hinweg entschieden zu haben. ${ }^{146}$

\section{Nach der "Aktion"}

Hitler beendete im August 1941 die „Aktion T4". Der letzte Transport aus Hall im August 1942 stellt für die Heil- und Pflegeanstalten im Deutschen Reich eine Ausnahme dar. Dass jedoch auch in anderen Anstalten nach dem Stopp der "offiziellen" Euthanasie nicht Schluss war mit dem Morden, beweisen die Sterberaten in den einzelnen Anstalten: Ab 1942 stiegen sie wieder enorm an. Inwieweit die InsassInnen der HPA Hall in Tirol davon betroffen waren, soll in diesem Kapitel erörtert werden. ${ }^{147}$

Dr. Hans Czermak bemühte sich - teils aus „blindem Gehorsam“ und Karrierebewusstsein, teils aus überzeugtem nationalsozialistischen Rassenwahn ${ }^{148}$ - ab November 1942 immer stärker, eine eigene Euthanasie-Station in Hall zu etablieren. Dazu kontaktierte er mehrmals Dr. Lonauer, der eine derartige Station einrichten und leiten sollte. ${ }^{149}$ Dieser Umstand kann als Beweis gelten, dass Dr. Klebelsberg sich davor sträubte, diesen letzten Schritt bei der Ermordung selbst Hand anzulegen, umzusetzen. ${ }^{150}$ Auch war es Klebelsberg, der nach eigenen Aussagen Czermak und Lonauer von derartigen Plänen abbrachte. Die Pläne lagen in den nächsten Jahren mehr oder weniger auf Eis, bis sich Lonauer im März 1945 hilfesuchend an Czermak wandte und ihn bat, ihm und seiner Familie eine geheime Unterkunft in Tirol zu beschaffen. Czermaks Antwort zeugt von seiner nationalsozialistischen Überzeugung bis zum Schluss: „Treten Sie, inkognito' vorübergehend als Oberarzt in unsere Heilanstalt Solbad Hall ein und organisieren Sie dort die Reduzierung des Krankenstandes, denn die Anstalt ist zum Bersten voll.."151 Ob Lonauer dem „Angebot" folgte, ist nicht belegt und gilt als unwahrscheinlich. ${ }^{152}$

\footnotetext{
146 Seifert, Sterben, S. 392-394.

147 Die folgende Darstellung fußt aus Mangel an zusätzlicher aktueller Literatur größtenteils auf Seifert, Sterben.

148 Schreiber, Idealist.

149 Seifert, Heil- und Pflegeanstalt, S. 116.

150 Ebd., S. 117, 143.

151 Hans Czermak an Rudolf Lonauer, 17.4.1945, abgedruckt in: Dokumentationsarchiv des österreichischen Widerstandes (Hrsg.), Widerstand und Verfolgung in Tirol 1934-1945. Band 1, Wien-München 1984, S. 497, zit. n. Seifert, Heil- und Pflegeanstalt, S. 117. 
Eindeutig nachgewiesen ist allerdings die stark erhöhte Sterblichkeit in der Anstalt zwischen 1943 und 1945. Lag die Anzahl der Todesfälle pro Jahr zwischen 1935 und 1938 bei durchschnittlich rund 4 Prozent, erhöhte sich die Zahl zwischen 1939 und 1943 auf 6,6 Prozent, und in den letzten beiden Kriegsjahren auf 17 (!) Prozent. ${ }^{153}$ Seifert macht dazu auf mehrere strukturelle, im Folgenden aufgelistete Bedingungen aufmerksam. ${ }^{154}$

\section{Die Versorgungslage}

Es liegt auf der Hand, dass die Versorgungslage im gesamten Reich in den letzten beiden Kriegsjahren miserabel war, und dass die Krise in der Beschaffung des Lebensnotwendigsten insbesondere die „unnützen Esser" in den Anstalten traf. Die Rationen an Lebensmitteln pro Person wurden drastisch gekürzt. 1946 machte sich die Lage besonders bemerkbar, als die Menschen mit einem Drittel dessen auskommen mussten, was in Friedenszeiten als normal galt. Dazu kam der Umstand, dass die InsassInnen der HPA Hall nicht über andere Formen der Lebensmittelbeschaffung, wie etwa den Handel über dem Schwarzmarkt, ihre Lage verbessern konnten. Zudem waren sie Opfer der nationalsozialistischen Politik, die auf eine grundsätzliche Benachteiligung von AnstaltsinsassInnen zielte. Dies bezeugt unter anderem ein Erlass von 1940: Von den den Krankenhäusern zugeteilten „Sonderzulagen“ waren die psychiatrischen Anstalten explizit ausgeschlossen. ${ }^{155}$ Der Erlass wurde erst im Mai 1946 in Tirol aufgehoben. Dass der Zustand der PatientInnen der HPA Hall nach Kriegsende sehr schlecht war, beweist die Aussage einer US-amerikanischen Sanitätseinheit: Diese habe nach Besichtigung der HPA Hall erklärt, ,in keinem Kazett [sic!] solcher Art herabgekommene Leute gefunden zu haben".156

Die desolate Versorgungssituation mit Brennstoffen in den Jahren 1944 und 1945 trugen nicht minder zu einer Verschlechterung der körperlichen Verfassung der InsassInnen bei, wenngleich davon die Bevölkerung als Ganzes gleichermaßen betroffen war und eine gezielte Benachteiligung der Anstalten bislang nicht nachgewiesen wurde. ${ }^{157}$

Zudem mangelte es nicht selten an lebensnotwendigen Medikamenten. Infektionskrankheiten breiteten sich auf den Stationen der HPA Hall rasant aus, und oftmals endeten viele der heute als harmlos geltenden Krankheiten mit dem Tod der/s Betroffenen.

Man kann davon ausgehen, dass diese markante Unterversorgung an Lebensmitteln, Heizstoffen und Medikamenten zum Tode vieler PatientInnen führte. Allerdings kann der Verdacht auf die gezielte Tötung beispielsweise durch Nahrungsentzug nicht be-

153 Die zugrunde legenden Zahlen beruhen auf Dirk Dunkel, Grundlegende statistische Auswertungen zur Heil- und Pflegeanstalt Hall während der NS-Zeit, in: Perz et al. (Hrsg.), Schlussbericht, S. 146.

154 Seifert, Heil- und Pflegeanstalt, S. 119-139.

155 Ebd., S. $120 \mathrm{f}$.

156 Protokoll über die mündliche Verhandlung der Disziplinarkommission für Landesbeamte beim Amte der Tiroler Landesregierung, 30.10.1951. TLA, ATLR, Präsisium (Personalakten) I, Personalakt Julius Vogt, zit. n. ebd., S. 124; diese Darstellung ist insofern noch erschreckender, als dass die für Hall zuständige 103. Infanteriedivision zuvor das KZ Dachau befreit hatte, ebd.

157 Ebd., S. $128 \mathrm{f}$. 
legt werden. ${ }^{158}$ Gleiches gilt für die Heizsituation ${ }^{159}$ und die Versorgung mit Medikamenten. ${ }^{160}$ Jedoch kann anhand der Quellen eine Art "Hierarchisierung des Wertes"161 der PatientInnen festgestellt werden, in dem Maße, als dass InsassInnen, die als heilbar galten, besser versorgt wurden als solche, bei denen eine Behandlung als aussichtslos angesehen wurde. ${ }^{162}$

Die räumliche Situation

Die ab 1940720 Betten der HPA Hall in Tirol waren bis 1944 durchgehend überbelegt. Die Transporte nach Hartheim und Niedernhart bedeuteten nur eine kleine Erleichterung, da den weggebrachten PatientInnen stets rasch neue folgten. Das hatte auch damit zu tun, dass nach 1940 vermehrt auch Südtirolerlnnen und Vorarlbergerlnnen nach Hall gebracht wurden. ${ }^{163}$ Besonders dramatisch war die Lage Mitte 1944, als für 702 PatientInnen nur insgesamt 640 Betten bereitstanden. Die hygienischen Bedingungen waren demnach katastrophal. Als extremes Beispiel dient die Darstellung der Männerabteilung 7: Hier waren Alte, Kranke und Pflegebedürftige untergebracht; zudem wurden nach Juni 1944 immer mehr neue "unruhige Patienten“164 hier einquartiert. Drei Viertel aller 72 nach Juni 1944 verstorbenen Patienten, die auf dem Anstaltsfriedhof beerdigt sind, starben hier. ${ }^{165}$ Alles in allem muss die räumliche Situation ähnlich katastrophale Zustände angenommen haben wie in Zeiten des Ersten Weltkrieges. ${ }^{166}$

\section{Personal}

Bereits 1937 waren in der HPA Hall in Tirol im Verhältnis zu anderen reichsdeutschen Anstalten wenige PflegerInnen und Ärzte aktiv: Der Pflegeschlüssel lag bei eins zu sieben, was bedeutet, dass auf eine/n Pflegerln sieben Patientlnnen kamen. Nach dem Anschluss wurde die Zahl der Pflegerlnnen erhöht. So kam es, dass in den ersten Kriegsjahren keine größeren Einbußen an Personal zu verzeichnen waren. Ab 1943 änderte sich die Lage aber radikal. Durch den Einzug zur Wehrmacht und Krankenstände verringerte sich die Anzahl der anwesenden Ärzte bei einer stetig wachsenden Zahl an InsassInnen. Kriegsbedingt kam es auch zu den weitaus folgenschwereren Engpässen bei den Pflegerlnnen, insbesondere bei den männlichen. ${ }^{167}$

158 Seifert, Heil- und Pflegeanstalt, S. 126.

159 Ebd., S. 129.

160 Seifert, Heil- und Pflegeanstalt, S. 132.

161 Christof Beyer, Die pfälzische Heil- und Pflegeanstalt in Klingenmünster im Nationalsozialismus, in: Arbeitskreis zur Erforschung der nationalsozialistischen „Euthanasie“ und Zwangssterilisation (Hrsg.), NS-_Euthanasie“ und lokaler Krankenmord in Oldenburg, Klingenmünster und Sachsen. Erinnerungskultur und Betroffenenperspektive (Berichte des Arbeitskreises 6), Münster 2011, S. 55-70, hier S. 57, zit. n. ebd., S. 126; dies war jedoch kein ausschließliches Phänomen der Kriegszeit, sondern bereits in den Jahrzehnten zuvor und unmittelbar nach 1945 mehr oder weniger gängige Praxis, siehe unter anderem Dietrich-Daum, Heil- und Pflegeanstalt, S. 105. 
Seifert führt dies mitunter als Grund für die höhere Sterberate bei Männern an. ${ }^{168}$ Die anthropologischen Studien zu den Leichenfunden auf dem ehemaligen Anstaltsfriedhof legen die Vermutung nahe, dass die PatientInnen vermehrt geschlagen wurden. ${ }^{169}$ Inwieweit dieses Vorgehen durch die Ideologie des Nationalsozialismus beeinflusst oder vielmehr damals gängige Praxis war, lässt sich nicht feststellen. Für Seifert ist eine Verbindung beider Motive am wahrscheinlichsten. ${ }^{170}$

Eine gezielte Tötung von InsassInnen mittels Medikamenten, wie sie nach Beendigung von "T4" in vielen reichsdeutschen Heil- und Pflegeanstalten üblich war, lässt sich für die HPA Hall nach derzeitigem Wissensstand weder beweisen, noch vollends widerlegen. ${ }^{171}$ Auch die Errichtung des Friedhofes im November 1942 steht wahrscheinlich nicht im Zusammenhang mit Czermaks Plänen, Morde vor Ort durchzuführen. Vielmehr erhoffte sich die Stadtverwaltung mehr Platz für den überfüllten Stadtfriedhof. ${ }^{172}$

Nichtsdestotrotz muss erwähnt werden, dass die Versorgungslage miserabel war. Die Ärzte der HPA Hall hatten sich offensichtlich mit der institutionalisierten Schlechterbehandlung der InsassInnen abgefunden. ${ }^{173}$ Der Tod stellte insofern einen ständigen Begleiter der PatientInnen der HPA Hall auch nach "T4" dar.

\section{Schluss}

Die nationalsozialistische Euthanasie stellt ein äußerst trauriges Kapitel der Zeitgeschichte dar. Dabei war die Rassenideologie, die der "Aktion“ zugrunde lag, keine reine Erfindung der Nazis. Sie bedienten sich scheinbar wissenschaftlicher Theorien und Konzepte, die dem Gedanken des Sozialdarwinismus der zweiten Hälfte des 19. Jahrhunderts entsprungen und bis in die 1940er-Jahre in immer perfideren Spielarten weiterentwickelt worden waren. Auch wenn die wissenschaftliche Disziplin der (deutschen) "Rassenhygiene" zu keiner Zeit deckungsgleich war mit dem politischen Programm der NSDAP, war doch sie es, die der Partei und vor allem Hitler ${ }^{174}$ die Ideen und die vorgeblich wissenschaftliche Grundlage gab. ${ }^{175}$

In einem weiteren, abstrakteren Sinn war es die Absicht des Verfassers zu veranschaulichen, wie die Wissenschaft (vor allem, wenn sie polemisch wirkt) langfristig das Denken und Handeln von Personen und Personengruppen beeinflussen kann. Der Nationalsozialismus und dessen institutionalisierter Massenmord waren zwar keine zwangsläufigen Folgen der zutiefst unmenschlichen Rassentheorien des 19. und 20.

168 Seifert, Heil- und Pflegeanstalt, S. 137.

169 George McGlynn/Nadine Carlichi-Witjes, Vorbericht zu den biologisch-anthropologischen Untersuchungen am Skelettmaterial des Friedhofs der Heil- und Pflegeanstalt Hall, in: Perz et al. (Hrsg.), Schlussbericht, S. 290-298 und im Besonderen ebd., S. 298.

170 Seifert, Heil- und Pflegeanstalt, S. $138 \mathrm{f}$.

171 Ebd., S. 143.

172 Oliver Seifert, Der Anstaltsfriedhof der Heil- und Pflegeanstalt Hall 1942-1945, in: Perz et al. (Hrsg.), Schlussbericht, S. $248 \mathrm{f}$.

173 Seifert, Heil- und Pflegeanstalt, S. 144.

174 Beispielsweise Hitler, Kampf, S. 311-362, bei dem unter anderem Rassenhygieniker und -theoretiker mit ihren Ideen Pate standen.

175 Weingart et al., Rasse, S. 370 
Jahrhunderts, aber deren Wesensverwandtschaft ist auch keineswegs als rein zufällig zu betrachten.

Die eigentliche „Aktion T4", die mit einem mündlichen Befehl Hitlers begann, lief unter großer Geheimhaltung. Für die Ermordung der „Ballastexistenzen“ wurde ein eigener Verwaltungsapparat mit bestimmten Vollmachten aufgebaut. Ein ausgeklügeltes Täuschungssystem sollte für die Geheimhaltung sorgen. Neben seiner radikalen Unmenschlichkeit sticht die „Aktion" vor allem auch wegen ihrer Rolle als „wichtige Vorstufe für den Holocaust“ „in organisatorischer, personeller und technologischer Hinsicht"176 hervor.

Mit dieser Arbeit konnte zudem gezeigt werden, dass aus dem Reichsgau Tirol-Vorarlberg mindestens 686 Personen (mindestens 466 aus Tirol, mindestens 220 aus Vorarlberg) in die Tötungsanstalten abtransportiert wurden. Die HPA Hall in Tirol erfüllte eine zentrale Sammelfunktion für den Reichsgau. Hier wurden die selektierten Personen aus den umliegenden Pflegeanstalten des Tiroler Raumes Reichsgaus konzentriert, ehe sie nach Hartheim oder Niedernhart weitertransportiert wurden. Die Vertreter des Reichsgaus waren besonders in der Person von Dr. Hans Czermak an der Ermordung von psychisch kranken und behinderten Menschen interessiert. Seine Unterwürfigkeit gegenüber dem NS-Regime sowie sein Karrierismus machten aus ihm einen der schärfsten Verfechter der "Tötung lebensunwerten Lebens". In der Rolle des Primars Klebelsberg wurde die ambivalente Haltung vieler Ärzte (sowie anderer Berufsgruppen) im Bezug mit dem Nationalsozialismus aufgezeigt. Obwohl er nach eigener Aussage ein Gegner der Euthanasie war, konnte er sich mit dem System arrangieren, um zumindest einen Teil der InsassInnen zu retten. Durch diese Praxis legitimierte er jedoch auch die Tötung vieler anderer PatientInnen.

\section{Literatur}

Ammon, Otto, Die Gesellschaftsordnung und ihre natürlichen Grundlagen. Entwurf einer Sozial-Anthropologie zum Gebrauch für alle Gebildeten, die sich mit sozialen Fragen befassen, Jena $1896^{2}$.

Baur, Erwin, Abriß der allgemeinen Variations- und Erblehre, in: Erwin Baur/Eugen Fischer/Fritz Lenz (Hrsg.), Menschliche Erblehre und Rassenhygiene (Eugenik). Band I: Menschliche Erblehre, München 1936', S. 1-94.

Becker, Peter Emil, Zur Geschichte der Rassenhygiene. Wege ins Dritte Reich, StuttgartNew York 1988.

Benz, Wolfgang, Geschichte des Dritten Reiches, Bonn 2010.

derStandard.at, Ausgrabungen in Hall in Tirol beendet, 14.11.2011, [http://derstandard. at/1319182805511/Psychiatrie-Friedhof-Ausgrabungen-in-Hall-in-Tirol-beendet], eingesehen 6.5.2014.

176 Neugebauer, NS-Euthanasieaktionen, S. 47. 
Projektteam „Psychiatrische Landschaften/Psichiatria Confini" (Hrsg.), Die unsichtbare Arbeit. Zur Geschichte der psychiatrischen Pflege im historischen Tirol von 1830 bis zur Gegenwart, in: Psychiatrische Landschaften | Psichiatria Confini, o. D., [http://arbeit. psychiatrische-landschaften.net/?page_id=6], eingesehen 5.10.2014.

Dietrich-Daum, Elisabeth, Die Psychiatrische Heil- und Pflegeanstalt Hall in Tirol 1830 bis 1940, in: Bertrand Perz/Thomas Albrich/Elisabeth Dietrich-Daum et al. (Hrsg.), Schlussbericht der Kommission zur Untersuchung der Vorgänge um den Anstaltsfriedhof des Psychiatrischen Krankenhauses in Hall in Tirol in den Jahren 1942 bis 1945 (Veröffentlichungen der Kommission zur Untersuchung der Vorgänge um den Anstaltsfriedhof des Psychiatrischen Krankenhauses in Hall in Tirol in den Jahren 1942 bis 1945), Innsbruck 2014, S. 99-110.

Duden online, Euthanasie, [http://www.duden.de/node/685056/revisions/1193702/ view], eingesehen 9.9.14.

Duden online, Eugenik, [http://www.duden.de/node/685056/revisions/1193702/ view], eingesehen 8.9.14.

Dunkel, Dirk, Grundlegende statistische Auswertungen zur Heil- und Pflegeanstalt Hall während der NS-Zeit, in: Bertrand Perz/Thomas Albrich/Elisabeth Dietrich-Daum et al. (Hrsg.), Schlussbericht der Kommission zur Untersuchung der Vorgänge um den Anstaltsfriedhof des Psychiatrischen Krankenhauses in Hall in Tirol in den Jahren 1942 bis 1945 (Veröffentlichungen der Kommission zur Untersuchung der Vorgänge um den Anstaltsfriedhof des Psychiatrischen Krankenhauses in Hall in Tirol in den Jahren 1942 bis 1945), Innsbruck 2014, S. 145-186.

Haeckel, Ernst, Natürliche Schöpfungsgeschichte. Gemeinverständliche wissenschaftliche Vorträge über die Entwicklungslehre im Allgemeinen und diejenige von Darwin, Goethe und Lamarck im Besonderen, über die Anwendung derselben, auf den Ursprung des Menschen und andere damit zusammenhängende Grundfragen der Naturwissenschaft, Berlin 1870², [http://babel.hathitrust.org/cgi/pt?id=hvd.3204406629 1840; view=1 up;seq=11], eingesehen 7.10.2014.

Hinterhuber, Hartmann, Ermordet und vergessen. Nationalsozialistische Verbrechen an psychisch Kranken und Behinderten in Nord- und Südtirol, Innsbruck 1995.

Hitler, Adolf, Mein Kampf, München $1939^{489}$.

Kammerhofer, Andrea, „Bis zum 1. September 1941 wurden desinfiziert: Personen: 70.273". Die „Hartheimer Statistik“, in: Brigitte Kepplinger/Gerhart Marckhgott/Hartmut Reese (Hrsg.), Tötungsanstalt Hartheim (Oberösterreich in der Zeit des Nationalsozialismus 3), Linz 201333, S. 117-130.

Kepplinger, Brigitte, NS-Euthanasie in Österreich. Die „Aktion T4" - Struktur und Ablauf, in: Brigitte Kepplinger/Gerhart Marckhgott/Hartmut Reese (Hrsg.), Tötungsanstalt Hartheim (Oberösterreich in der Zeit des Nationalsozialismus 3), Linz 2013³, S. 35-62. 
Dies., Tötungsanstalt Hartheim 1940-1945, in: Brigitte Kepplinger/Gerhart Marckhgott/ Hartmut Reese (Hrsg.), Tötungsanstalt Hartheim (Oberösterreich in der Zeit des Nationalsozialismus 3), Linz 2013³, S. 63-116.

Dies., Zum Gesundheitssystem im Reichsgau Tirol-Vorarlberg. Eine Skizze, in: Bertrand Perz/Thomas Albrich/Elisabeth Dietrich-Daum et al. (Hrsg.), Schlussbericht der Kommission zur Untersuchung der Vorgänge um den Anstaltsfriedhof des Psychiatrischen Krankenhauses in Hall in Tirol in den Jahren 1942 bis 1945 (Veröffentlichungen der Kommission zur Untersuchung der Vorgänge um den Anstaltsfriedhof des Psychiatrischen Krankenhauses in Hall in Tirol in den Jahren 1942 bis 1945), Innsbruck 2014, S. 83-89.

Dies./Reese, Hartmut, Die Nationalsozialistische Euthanasieverbrechen. Orte und „Aktionen“, in: Brigitte Kepplinger/Gerhart Marckhgott/Hartmut Reese (Hrsg.), Tötungsanstalt Hartheim (Oberösterreich in der Zeit des Nationalsozialismus 3), Linz 2013³, S. 437-473.

Klee, Ernst, „Euthanasie“ im Dritten Reich. Die „Vernichtung lebensunwerten Lebens" (Die Zeit des Nationalsozialismus 18674), Frankfurt am Main 2010.

Klepsch, Thomas, Nationalsozialistische Ideologie. Eine Beschreibung ihrer Struktur vor 1933 (Studien zum Nationalsozialismus 2), Münster 1990.

Leimgruber, Florian, Euthanasie und Sterilisierung im ehemaligen „Reichsgau Tirol-Vorarlberg" während des II. Weltkrieges 1939-45, Diss. Innsbruck [1988].

Mayer, Thomas, Familie, Rasse und Genetik. Deutschnationale Eugeniken im Österreich der Zwischenkriegszeit, in: Gerhard Baader/Veronika Hofer/Thomas Mayer (Hrsg.), Eugenik in Österreich. Biopolitische Strukturen von 1900-1945, Wien 2007, S. 162-183.

McGlynn, George/Carlichi-Witjes, Nadine, Vorbericht zu den biologisch-anthropologischen Untersuchungen am Skelettmaterial des Friedhofs der Heil- und Pflegeanstalt Hall, in: Bertrand Perz/Thomas Albrich/Elisabeth Dietrich-Daum et al. (Hrsg.), Schlussbericht der Kommission zur Untersuchung der Vorgänge um den Anstaltsfriedhof des Psychiatrischen Krankenhauses in Hall in Tirol in den Jahren 1942 bis 1945 (Veröffentlichungen der Kommission zur Untersuchung der Vorgänge um den Anstaltsfriedhof des Psychiatrischen Krankenhauses in Hall in Tirol in den Jahren 1942 bis 1945), Innsbruck 2014, S. 275-299.

Neugebauer, Wolfgang, Die „Aktion T4", in: Kepplinger et al., (Hrsg.), Tötungsanstalt Hartheim (Oberösterreich in der Zeit des Nationalsozialismus 3), Linz 201333, S. 17-34.

Ders., NS-Euthanasieaktionen in Österreich. Ein Überblick, in: Bertrand Perz/Thomas Albrich/Elisabeth Dietrich-Daum et al. (Hrsg.), Schlussbericht der Kommission zur Untersuchung der Vorgänge um den Anstaltsfriedhof des Psychiatrischen Krankenhauses in Hall in Tirol in den Jahren 1942 bis 1945 (Veröffentlichungen der Kommission zur Untersuchung der Vorgänge um den Anstaltsfriedhof des Psychiatrischen Krankenhauses in Hall in Tirol in den Jahren 1942 bis 1945), Innsbruck 2014, S. 35-48. 
Parma.Repubblica.it, [http://da-parma-a-mauthausen-parma.blogautore.repubblica. it/], eingesehen 5.10.2014.

Schmuhl, Hans-Walter, Rassenhygiene, Nationalsozialismus, Euthanasie. Von der Verhütung zur Vernichtung „lebensunwerten Lebens“, 1890-1945 (Kritische Studien zur Geschichtswissenschaft 75), Göttingen 1987.

Schreiber, Horst, Ein „Idealist, aber kein Fanatiker"? Dr. Hans Czermak und die NS-Euthanasie in Tirol, 2008, [http://www.horstschreiber.at/texte/czermak-und-die-nseuthanasie-in-tirol], eingesehen 19.9.2014.

Ders., Tiroler Studien zu Geschichte und Politik, Bd. 8: Nationalsozialismus und Faschismus in Tirol und Südtirol. Opfer, Täter, Gegner, Innsbruck 2008.

Schwarz, Peter, Der Gerichtsakt Georg Renno als Quelle für das Projekt Hartheim, in: Zentrale österreichische Forschungsstelle Nachkriegsjustiz (Hrsg.), Nachkriegsjustiz. at, 11.9.2014, [http://www.nachkriegsjustiz.at/ns_verbrechen/euthanasie/renno_psw. php\#zw3], eingesehen 5.10.2014.

Seifert, Oliver, "Sterben hätten sie auch hier können“. Die „Euthanasie“-Transporte aus der Heil- und Pflegeanstalt Hall in Tirol nach Hartheim und Niedernhart, in: Brigitte Kepplinger/Gerhart Marckhgott/Hartmut Reese (Hrsg.), Tötungsanstalt Hartheim (Oberösterreich in der Zeit des Nationalsozialismus 3), Linz 2013³, S. 359-410.

Ders., Das Sterben in der Heil- und Pflegeanstalt Hall 1942-1945, in: Bertrand Perz/ Thomas Albrich/Elisabeth Dietrich-Daum et al. (Hrsg.), Schlussbericht der Kommission zur Untersuchung der Vorgänge um den Anstaltsfriedhof des Psychiatrischen Krankenhauses in Hall in Tirol in den Jahren 1942 bis 1945 (Veröffentlichungen der Kommission zur Untersuchung der Vorgänge um den Anstaltsfriedhof des Psychiatrischen Krankenhauses in Hall in Tirol in den Jahren 1942 bis 1945), Innsbruck 2014, S. 111-144.

Ders., Der Anstaltsfriedhof der Heil- und Pflegeanstalt Hall 1942-1945, in: Bertrand Perz/ Thomas Albrich/Elisabeth Dietrich-Daum et al. (Hrsg.), Schlussbericht der Kommission zur Untersuchung der Vorgänge um den Anstaltsfriedhof des Psychiatrischen Krankenhauses in Hall in Tirol in den Jahren 1942 bis 1945 (Veröffentlichungen der Kommission zur Untersuchung der Vorgänge um den Anstaltsfriedhof des Psychiatrischen Krankenhauses in Hall in Tirol in den Jahren 1942 bis 1945), Innsbruck 2014, S. 229-249.

Ders., Dr. Ernst Klebelsberg, in: Projektteam „Psychiatrische Landschaften/Psichiatria Confini" (Hrsg.), Psychiatrische Landschaften | Psichiatria Confini, o. D. [http://psychiat rische-landschaften.net/node/146], eingesehen 5.10.2014.

Stepanek, Friedrich, Zur Untersuchung des Personals der Heil- und Pflegeanstalt Hall, in: Bertrand Perz/Thomas Albrich/Elisabeth Dietrich-Daum et al. (Hrsg.), Schlussbericht der Kommission zur Untersuchung der Vorgänge um den Anstaltsfriedhof des Psychiatrischen Krankenhauses in Hall in Tirol in den Jahren 1942 bis 1945 (Veröffentlichungen der Kommission zur Untersuchung der Vorgänge um den Anstaltsfriedhof des Psychiatrischen Krankenhauses in Hall in Tirol in den Jahren 1942 bis 1945), S. 187-209. 
Weingart, Peter/Kroll, Jürgen/Bayertz, Kurt, Rasse, Blut und Gene. Geschichte der Eugenik und Rassenhygiene in Deutschland, Frankfurt am Main 1988.

Zischka, Johannes, Die NS-Rassenideologie. Machttaktisches Instrument oder handlungsbestimmendes Ideal? (Geschichte und ihre Hilfswissenschaften 274), Frankfurt am Main-Bern-New York 1986.

\section{Abkürzungsverzeichnis}

$\begin{array}{ll}\text { ATLR } & \text { Amt der Tiroler Landesregierung } \\ \text { DGR } & \text { Deutsche Gesellschaft für Rassenhygiene } \\ \text { Gekrat } & \text { Gemeinnützige Krankentransport GmbH } \\ \text { GzVeN } & \begin{array}{l}\text { Gesetz zur Verhütung erbkranken Nachwuchses, bzw. Erbgesund- } \\ \text { heitsgesetz } \\ \text { HHStA }\end{array} \quad \text { Hessisches Hauptstaatsarchiv } \\ \text { HPA } & \text { Heil- und Pflegeanstalt } \\ \text { KdF } & \text { Kanzlei des Führers } \\ \text { LG } & \text { Land(es)gericht } \\ \text { RAG } & \text { Reichsarbeitsgemeinschaft Heil- und Pflegeanstalten } \\ \text { RMdl } & \text { Reichsstatthalterei } \\ \text { RStH } & \text { Staatsanwaltschaft } \\ \text { StA } & \text { Tiroler Landesarchiv } \\ \text { TLA } & \text { Wiener Gesellschaft für Rassenpflege (Rassenhygiene) } \\ \text { WGR } & \end{array}$

\section{T4-Personenverzeichnis ${ }^{177}$}

Allers, Dietrich

Bohne, Gerhard

Bouhler, Philipp

Brack, Viktor
Geschäftsführer der Zentraldienststelle (T4), † 22.3.1975 München.

1939 bis 1940 Leiter der Reichsarbeitsgemeinschaft für Heil- und Pflegeanstalten (RAG), † 8.7.1981.

Leiter der Kanzlei des Führers, ab 1939 "Euthanasiebevollmächtigter", † 19.5.1945 Dachau (Suizid).

Oberdienstleiter des Hauptamtes II der Kanzlei des Führers in Berlin (mit „Aktion T4" beauftragt), † 2.6.1948

177 Die Darstellung entstammt aus verschiedenen Werken der angegebenen Literatur; als größte Hilfe für den Verfasser dieser Arbeit sowie als Empfehlung für weitere Personenrecherchen im Zusammenhang mit der Aktion T4 soll auf das äußerst umfangreiche Verzeichnis in Klee, Euthanasie, S. 544-605 verwiesen werden. 
Prof. Dr. Brandt, Karl

Dr. Czermak, Hans
Landsberg (Hinrichtung).

Adolf Hitlers chirurgischer Begleitarzt, ab 1939 "Euthanasiebevollmächtigter", † 2.6.1948 Landsberg (Hinrichtung).

Leiter der Abteilung III ("Volkspflege“) im Gau Tirol-Vorarlberg, † 30.4.1975 Innsbruck.

Im Original ist hier ein Foto von Dr. Hans Czermak zusammen mit Gauleiter Franz Hofer und Volksgruppenführer Peter Hofer aus dem Juni 1941 abgebildet.
Denz, Egon

Prof. Dr. Heyde, Werner

Hofer, Franz

Dr. Jekelius, Erwin

Dr. Klebelsberg, Ernst
1938 bis 1945 Oberbürgermeister von Innsbruck, Mitglied der NSDAP und der SS, † 15.12.1979 Innsbruck.

bis 1941 Leiter der medizinischen Abteilung der Zentraldienststelle („T4"), † 13.2.1964 Zuchthaus Butzbach (Suizid).

Gauleiter in Tirol-Vorarlberg, † 18.2.1975 Mülheim an der Ruhr.

T4-Gutachter, 1940 bis 1942 Leiter der "Kinderfachabteilung"Wien-Spiegelgrund, † 8.5.1952 Vladimirski Gefängnis des sowjetischen Innenministeriums.

von 1925 bis 1950 Primararzt in der Heil- und Pflegeanstalt Hall in Tirol, + 13.5.1957 Hall in Tirol.
Dr. Linden, Herbert

Dr. Lonauer, Rudolf
Ministerialdirigent im Reichsministerium des Innern, Reichsbeauftragter für die Heil- und Pflegeanstalten, † 27.4.1945 Berlin (Suizid).

Anstaltsleiter der Heil- und Pflegeanstalt Niedernhart bei Linz und ärztlicher Leiter der Tötungsanstalt Hartheim, zudem Beauftragter der Reichsarbeitsgemeinschaft für Heil- und Pflegeanstalten, und "T4"-Gutachter, + 5.5.1945 Linning bei Neuhofen a. d. Krems (Suizid).

Gauamtsleiter für Volksgesundheit im Gau Tirol-Vorarlberg, † k. A. 
Dr. Mennecke, Friedrich

Prof. Dr. Nitsche, H. Paul

Nohel, Vinzenz

Dr. Renno, Georg
„T4"-Gutachter, † 28.1.1947 Zuchthaus Butzbach.

ab 1941 Leiter der medizinischen Abteilung der Zentraldienststelle („T4"), † 25.3.1947 Dresden (Hinrichtung).

Leichenverbrenner in der Tötungsanstalt Hartheim, † 27.5.1946 Landsberg (Hinrichtung).

Stellvertreter von Lonauer in Hartheim, † 4.10.1997 Neustadt an der Weinstraße.

Im Original ist hier ein Foto von Dr. Georg

Renno abgebildet.

Dr. Scharfetter, Helmut

Vorberg, Reinhold
Vorstand der Psychiatrischen Klinik Innsbruck, SS-Untersturmführer, Mitglied der NSDAP und des NS-Ärztebundes, + 3.6.1979.

Leiter der Gemeinnützigen Krankentransport GmbH (Gekrat), † 2.10.1983 Bonn.

Thomas Walli ist Studierender des Bachelorstudiums Geschichte im 9. Semester und des Masterstudiums Politikwissenschaft im 1. Semester an der Universität Innsbruck. thomas.walli@student.uibk.ac.at

\section{Zitation dieses Beitrages}

Thomas Walli, „Wir kommen unter die Metzger.” Die Umsetzung des nationalsozialistischen Euthanasieprogramms im Reichsgau Tirol-Vorarlberg, in: historia.scribere 8 (2016), S. 253-286, [http://historia.scribere.at], 2015-2016, eingesehen 14.6.2016 (=aktuelles Datum).

Creative Commons Licences 3.0 Österreich unter Wahrung der Urheberrechte der Autorlnnen. 